\title{
Strong Isospin Breaking of the Nucleon and Delta Masses on the Lattice
}

\author{
Brian C. Tiburzi ${ }^{1, *}$ and André Walker-Loud ${ }^{2, \text {, }}$ \\ ${ }^{1}$ Department of Physics Box 90305, Duke University, Durham, NC 27708-0305 \\ ${ }^{2}$ Department of Physics Box 351560, University of Washington, Seattle, WA 98195-1560
}

(Dated: August 29, 2018)

\begin{abstract}
Strong isospin breaking in the spectrum of the nucleons and deltas can be studied in lattice QCD with the help of chiral perturbation theory. At leading order in the chiral expansion, the mass splittings between the proton and neutron and between the deltas are linear in the quark mass difference. The next-to-leading order contributions to these splittings vanish even away from the strong-isospin limit. Therefore, any non-linear quark mass dependence of these mass splittings is a signal of the next-to-next-to-leading order mass contributions, thus providing access to LECs at this order. We determine the mass splittings of the nucleons and deltas in two-flavor, heavy baryon chiral perturbation theory to next-to-next-to-leading order. We also derive expressions for the nucleon and delta masses in partially quenched chiral perturbation theory to the same order. The resulting mass expressions will be useful both for the extrapolation of lattice data on baryon masses, and for the study of strong isospin breaking.
\end{abstract}

PACS numbers: 12.38.Gc, 12.39.Fe

\section{INTRODUCTION}

Understanding nuclear physics from first principles is a long standing challenge. Quantum chromodynamics (QCD) is the well established theory of strong interactions that describes the underlying interactions of colored quarks and gluons which bind together to form the color neutral hadrons observed in nature: nucleons, pions, etc. The equations of motion for the quarks and gluons are non-linear, and at energy scales relevant for nuclear physics, $E \sim 1 \mathrm{GeV}$, the theory is non-perturbative. Thus the description of hadrons from first principles remains elusive. One can write down an effective theory of QCD, chiral perturbation theory $(\chi \mathrm{PT})[\underline{1}$, which provides a model-independent description of the low energy interactions of the hadrons by preserving the symmetries of QCD. $\chi \mathrm{PT}$ is written in terms of the pions (pseudo-Goldstone bosons which appear from spontaneous chiral symmetry breaking) and their interactions with baryonic states, like the nucleons and delta-resonances. As the pions are the lightest particles in the theory, they dominate the low-energy interactions of the hadrons. In $\chi \mathrm{PT}$, short distance physics is encoded in an infinite tower of higher dimensional operators, whose coefficients are low-energy constants (LECs). The utility of $\chi \mathrm{PT}$ is that these higher dimensional operators are suppressed by appropriate powers of the cutoff, where operators with higher inverse powers of the cutoff are higher order in the expansion. Thus, to a given order, one needs to keep only a finite number of operators, and the theoretical error associated with a given calculation is indicated by the size of omitted terms. Symmetry constrains the number of operators which enter at a given order, but not the numerical values of their coefficients. Thus the LECs must be fit by comparing either to experiment or lattice QCD.

Lattice QCD is a tool one can use to numerically calculate QCD observables from first principles - the mass spectrum of baryons and mesons being notable examples. Due to computational restrictions, however, the light quarks must presently be simulated with unphysically large masses. So that observables calculated on the lattice can be extrapolated from the larger quark masses used in lattice simulations to the the physical quark masses, one requires an understanding of the quark mass dependence of QCD. For low-energy QCD, $\chi \mathrm{PT}$ is precisely what is needed, as the quark masses explicitly break chiral symmetry. For the up and down quarks, this breaking can be treated perturbatively; thus, these quark masses enter $\chi \mathrm{PT}$ as parameters of the theory, allowing QCD observables to be calculated as a systematic expansion in powers of the quark masses. Consequently lattice QCD can be used in conjunction with $\chi \mathrm{PT}$ to first determine the LECs relevant to a given observable, and once these LECs are determined, extrapolate to make rigorous first principles predictions of QCD observables.

Lattice QCD calculations of the baryon masses began in the early 1980's but until recently, unquenched lattice calculations have been limited to quark masses such that the pion has been rather heavy, $m_{\pi} \geq 500 \mathrm{MeV}[2,3$, , 4, 5, , 6, 7, 8, 9, 10]. There are a handful of fits to the lattice data on baryon masses using $\chi \mathrm{PT}$ 11, 12, 13, 14]. However, the 
viability of $\chi \mathrm{PT}$ with such large pion masses is questionable [15, 16]. Ideally, one would like to see lattice data with all the pion masses less than $\sim 400 \mathrm{MeV}$, to properly map out the chiral regime. This is a very exciting time, as we are beginning to see lattice data of baryonic observables with $m_{\pi} \sim 330 \mathrm{MeV}$, e.g. [9]. If not the pions accessible today, then the ones in the near future will be light enough that one can apply chiral perturbation theory to observables calculated from lattice QCD. We anticipate that lattice QCD in conjunction with $\chi \mathrm{PT}$ and PQ $\chi \mathrm{PT}$ will soon be making predictions of nucleon observables with theoretical errors at the $20 \%$ level [16]. ${ }^{1}$

Calculation of the baryonic mass spectrum from lattice QCD data in the chiral regime will be a first step in understanding nuclear physics from first principles. To this end, we determine the masses of the nucleons and delta-resonances to next-to-next-to-leading order (NNLO) in two-flavor non-degenerate $\chi \mathrm{PT}$. We highlight the mass splittings among the nucleons and deltas, as they provide a way to determine some of the LECs entering at NNLO. This is because strong isospin breaking in the baryon spectrum receives the first non-linear quark mass dependence at NNLO. In the appendix we calculate the masses of the nucleons and deltas in PQ $\chi \mathrm{PT}$, with two non-degenerate valence and sea quarks. These expressions are necessary to extrapolate partially quenched lattice data of the nucleon and delta masses to the physical values of the quark masses.

\section{MASSES IN $\chi \mathbf{P T}$}

In this section, we calculate the masses of the nucleons and deltas in the non-degenerate $\mathrm{SU}(2)$ flavor group. We start by reviewing the chiral Lagrangian for mesons and baryons. Next we calculate the masses of the nucleons and finally the masses of the deltas. The masses of the nucleons and deltas have been investigated considerably in $\chi \mathrm{PT}$, see e.g. 17, 18, 19, 20, 21, 22, 23, 24, 25, 26, 27, 28]. In Appendix @ we take up the partially quenched calculation.

\section{A. $\chi \mathbf{P T}$ Lagrangian}

For two flavors of massless quarks, the QCD Lagrangian exhibits a chiral symmetry, $\mathrm{SU}(2)_{L} \otimes \mathrm{SU}(2)_{R} \otimes \mathrm{U}(1)_{V}$. This symmetry is spontaneously broken by the vacuum down to $\mathrm{SU}(2)_{V} \otimes \mathrm{U}(1)_{V}$. $\chi \mathrm{PT}$ is the effective theory generated by expanding about the physical vacuum state of QCD. Without the source of explicit chiral symmetry breaking generated by the quark mass term, the pions would appear as the Goldstone bosons of this symmetry breaking. In reality the up and down quark masses are not zero but are quite small compared to the scale of chiral symmetry breaking. Thus the pions appear in nature as pseudo-Goldstone bosons.

To build the effective theory, we collect the pseudo-Goldstone bosons in an exponential matrix

$$
\Sigma=\exp \left(\frac{2 i \Phi}{f}\right)=\xi^{2}, \quad \Phi=\left(\begin{array}{cc}
\frac{1}{\sqrt{2}} \pi^{0} & \pi^{+} \\
\pi^{-} & -\frac{1}{\sqrt{2}} \pi^{0}
\end{array}\right) .
$$

The pion decay constant $f$ is $132 \mathrm{MeV}$ in the above conventions. The effective Lagrangian describing the dynamics of the pions at leading order in $\chi \mathrm{PT}$ is [1]

$$
\mathfrak{L}=\frac{f^{2}}{8} \operatorname{tr}\left(\partial^{\mu} \Sigma^{\dagger} \partial_{\mu} \Sigma\right)+\lambda \operatorname{tr}\left(m_{q}^{\dagger} \Sigma+m_{q} \Sigma^{\dagger}\right) .
$$

Above the quark mass matrix $m_{Q}$ is given by

$$
m_{q}=\operatorname{diag}\left(m_{u}, m_{d}\right) .
$$

Expanding the Lagrangian Eq. (2) one finds that to leading order the pions are canonically normalized with their masses given by

$$
m_{\pi}^{2}=\frac{4 \lambda}{f^{2}}\left(m_{u}+m_{d}\right)
$$

\footnotetext{
${ }^{1}$ We mean that the $\chi \mathrm{PT}$ and PQ $\chi \mathrm{PT}$ expressions will be fit only to lattice calculations but not experimental values. These expressions will then be used to extrapolate down to the physical quark masses, make predictions of experimental observables, and ultimately predictions of observables not currently accessible via experiment.
} 
The baryons are three quark states in the spectrum of QCD. To include the lowest-lying spin- $\frac{1}{2}$ and spin- $\frac{3}{2}$ baryons into $\chi \mathrm{PT}$, we use heavy baryon $\chi \mathrm{PT}(\mathrm{HB} \chi \mathrm{PT})[18,29,30,31,32,33]$. As is well known, this approach leads to a consistent power-counting scheme for including the baryons. In $\mathrm{SU}(2)$, there is a doublet $N$ of spin- $\frac{1}{2}$ nucleons

$$
N=\left(\begin{array}{l}
p \\
n
\end{array}\right)
$$

and a quartet of spin- $\frac{3}{2}$ deltas. These latter states are contained in the completely symmetric tensor $T^{i j k}$, where

$$
T^{111}=\Delta^{++}, \quad T^{112}=\frac{1}{\sqrt{3}} \Delta^{+}, \quad T^{122}=\frac{1}{\sqrt{3}} \Delta^{0}, \quad \text { and } \quad T^{222}=\Delta^{-} .
$$

The free Lagrangian for nucleons and deltas to leading order in HB $\chi \mathrm{PT}$ can be written as

$$
\begin{aligned}
\mathfrak{L}= & i \bar{N} v \cdot D N+2 \alpha_{M} \bar{N} \mathcal{M} N+2 \sigma_{M} \bar{N} N \operatorname{tr}(\mathcal{M}) \\
& -i \bar{T}^{\mu} v \cdot D T_{\mu}+\Delta \bar{T}^{\mu} T_{\mu}+2 \gamma_{M} \bar{T}^{\mu} \mathcal{M} T_{\mu}-2 \bar{\sigma}_{M} \bar{T}^{\mu} T_{\mu} \operatorname{tr}(\mathcal{M}) .
\end{aligned}
$$

Above, $\Delta$ is the leading-order mass splitting between the nucleons and deltas in the chiral limit. As a dimensionful parameter it must be treated in the power counting and we treat $\Delta \sim \mathcal{O}(q)$ 34, 35]. The chirally covariant derivative $D^{\mu}$ acts on the nucleon field as

$$
\left(D^{\mu} N\right)_{i}=\partial^{\mu} N_{i}+\left(V^{\mu}\right)_{i}^{i^{\prime}} N_{i^{\prime}}
$$

and on the delta field as

$$
\left(D^{\mu} T\right)_{i j k}=\partial^{\mu} T_{i j k}+\left(V^{\mu}\right)_{i}^{i^{\prime}} T_{i^{\prime} j k}+\left(V^{\mu}\right)_{j}{ }^{\prime} T_{i j^{\prime} k}+\left(V^{\mu}\right)_{k}{ }^{k^{\prime}} T_{i j k^{\prime}}
$$

The vector and axial-vector meson fields are given by

$$
V_{\mu}=\frac{1}{2}\left(\xi \partial_{\mu} \xi^{\dagger}+\xi^{\dagger} \partial_{\mu} \xi\right), \quad A_{\mu}=\frac{i}{2}\left(\xi \partial_{\mu} \xi^{\dagger}-\xi^{\dagger} \partial_{\mu} \xi\right)
$$

and finally the spurion mass field

$$
\mathcal{M}=\frac{1}{2}\left(\xi^{\dagger} m_{q} \xi^{\dagger}+\xi m_{q}^{\dagger} \xi\right)
$$

The Lagrange density describing the interactions of the nucleons and deltas with the pions at leading order can be written as

$$
\mathfrak{L}=2 g_{A} \bar{N} S \cdot A N+g_{\Delta N}\left(\bar{T}^{\mu} A_{\mu} N+\bar{N} A^{\mu} T_{\mu}\right)+2 g_{\Delta \Delta} \bar{T}^{\mu} S \cdot A T_{\mu},
$$

with $S^{\mu}$ as the covariant spin operator. Additionally we need higher-order terms to calculate masses at NNLO. The first set of higher-order terms are all operators that are constrained by reparameterization invariance [36, 37]. These terms must be included to insure the Lorentz invariance of $\mathrm{HB} \chi \mathrm{PT}$ at $\mathcal{O}\left(1 / M_{B}\right)^{2}$, where $M_{B} \sim \Lambda_{\chi}$ is the average nucleon mass in the chiral limit. As a result of reparameterization invariance, these operators have fixed coefficients and explicitly these operators are

$$
\begin{aligned}
\mathfrak{L}= & -\bar{N} \frac{D_{\perp}^{2}}{2 M_{B}} N+\bar{T}^{\mu} \frac{D_{\perp}^{2}}{2 M_{B}} T_{\mu}+g_{A}\left(\bar{N} \frac{i \overleftarrow{D} \cdot S}{M_{B}} v \cdot A N-\bar{N} v \cdot A \frac{S \cdot i \vec{D}}{M_{B}} N\right) \\
& +g_{\Delta \Delta}\left(\bar{T}^{\mu} \frac{i \overleftarrow{D} \cdot S}{M_{B}} v \cdot A T_{\mu}-\bar{T}^{\mu} v \cdot A \frac{S \cdot i \vec{D}}{M_{B}} T_{\mu}\right)
\end{aligned}
$$

\footnotetext{
${ }^{2}$ For an earlier, alternate approach for the nucleons, see, e.g., Ref. 32].
} 
where $D_{\perp}^{2}=D^{2}-(v \cdot D)^{2}$.

Next there are three sets of higher-dimensional operators whose coefficients are not pre-determined. The first set makes contributions to the mass of the nucleons at NNLO. The operators in this set are

$$
\begin{gathered}
\mathfrak{L}=\frac{1}{4 \pi f}\left\{b_{1}^{M} \bar{N} \mathcal{M}_{+}^{2} N+b_{5}^{M} \bar{N} N \operatorname{tr}\left(\mathcal{M}_{+}^{2}\right)+b_{6}^{M} \bar{N} \mathcal{M} N \operatorname{tr}(\mathcal{M})+b_{8}^{M} \bar{N} N[\operatorname{tr}(\mathcal{M})]^{2}\right. \\
\left.+b^{A} \bar{N} N \operatorname{tr}(A \cdot A)+b^{v A} \bar{N} N \operatorname{tr}(v \cdot A v \cdot A)\right\} .
\end{gathered}
$$

The LECs $b_{i}^{M}, b^{A}$, and $b^{v A}$ are all dimensionless. The choice in numbering the coefficients was made to be consistent with [26], as will become clear in Appendix \$] where we address the partially quenched calculation. The second set of higher-dimensional operators with undetermined coefficients consists of operators that contribute to the delta masses at NNLO. These operators are

$$
\begin{aligned}
& \mathfrak{L}=\frac{1}{4 \pi f}\left\{t_{2}^{A} \bar{T}_{\mu}^{k j i}\left(A_{\nu}\right)_{i}^{i^{\prime}}\left(A^{\nu}\right)_{j}^{j^{\prime}} T_{i^{\prime} j^{\prime} k}^{\mu}+t_{3}^{A}\left(\bar{T}_{\mu} T^{\mu}\right) \operatorname{tr}\left(A_{\nu} A^{\nu}\right)\right. \\
& +t_{2}^{\tilde{A}} \bar{T}_{\mu}^{k j i}\left(A^{\mu}\right)_{i} i^{\prime}\left(A_{\nu}\right)_{j}{ }^{j^{\prime}} T_{i^{\prime} j^{\prime} k}^{\nu}+t_{3}^{\tilde{A}} \bar{T}_{\mu} T^{\nu} \operatorname{tr}\left(A^{\mu} A_{\nu}\right) \\
& +t_{2}^{v A} \bar{T}_{\mu}^{k j i}(v \cdot A)_{i} i^{\prime}(v \cdot A)_{j}{ }^{j^{\prime}} T_{i^{\prime} j^{\prime} k}^{\mu}+t_{3}^{v A} \bar{T}_{\mu} T^{\mu} \operatorname{tr}(v \cdot A v \cdot A) \\
& +t_{1}^{M} \bar{T}_{\mu}^{k j i}(\mathcal{M M})_{i} i^{i^{\prime}} T_{i^{\prime} j k}^{\mu}+t_{2}^{M} \bar{T}_{\mu}^{k j i}(\mathcal{M})_{i}{ }^{i^{\prime}}(\mathcal{M})_{j}{ }^{j^{\prime}} T_{i^{\prime} j^{\prime} k}^{\mu}+t_{3}^{M} \bar{T}_{\mu} T^{\mu} \operatorname{tr}(\mathcal{M M}) \\
& \left.+t_{4}^{M}\left(\bar{T}_{\mu} \mathcal{M} T^{\mu}\right) \operatorname{tr}(\mathcal{M})+t_{5}^{M} \bar{T}_{\mu} T^{\mu}[\operatorname{tr}(\mathcal{M})]^{2}\right\} \text {, }
\end{aligned}
$$

and all of the LECs $t_{i}^{M}, t_{i}^{A}, t_{i}^{v A}$, and $t_{i}^{\tilde{A}}$ are dimensionless.

The last set of higher-dimensional operators with undetermined coefficients all involve the mass-splitting parameter $\Delta$, which is a singlet under chiral transformations. Including the spin- $\frac{3}{2}$ fields in $\chi \mathrm{PT}$ thus requires the addition of operators involving powers of $\Delta / \Lambda_{\chi}$. We shall not write out such operators explicitly. To account for the effects of these operators, all LECs in the calculation must be treated as arbitrary polynomial functions of $\Delta / \Lambda_{\chi}$ and expanded out to the required order. For example

$$
\begin{aligned}
\alpha_{M} \rightarrow \alpha_{M}\left(\frac{\Delta}{\Lambda_{\chi}}\right) & =\alpha_{M}\left(1+\alpha_{1} \frac{\Delta}{\Lambda_{\chi}}+\alpha_{2} \frac{\Delta^{2}}{\Lambda_{\chi}^{2}}\right), \\
\gamma_{M} \rightarrow \gamma_{M}\left(\frac{\Delta}{\Lambda_{\chi}}\right) & =\gamma_{M}\left(1+\gamma_{1} \frac{\Delta}{\Lambda_{\chi}}+\gamma_{2} \frac{\Delta^{2}}{\Lambda_{\chi}^{2}}\right), \\
g_{A} \rightarrow g_{A}\left(\frac{\Delta}{\Lambda_{\chi}}\right) & =g_{A}\left(1+g_{1} \frac{\Delta}{\Lambda_{\chi}}\right) .
\end{aligned}
$$

Determination of these LECs requires the ability to tune the parameter $\Delta$ and for this reason we do not keep such operators explicitly. Furthermore at this order the nucleon mass, $M_{0}$, and the nucleon-delta mass splitting in the chiral limit are also functions of $\Delta / \Lambda_{\chi}$. This variation, however, can be absorbed into a redefinition of the parameters $M_{0}$, and $\Delta$ (including contributions from loop graphs). We use these value for $M_{0}$ and $\Delta$ everywhere. Differences resulting from using this value of $\Delta$ in expressions for loop graphs are beyond the order we work.

\section{B. Nucleons}

The mass of the $i^{\text {th }}$ nucleon in the chiral expansion can be written as

$$
M_{B_{i}}=M_{0}(\Delta)-M_{B_{i}}^{(1)}(\mu, \Delta)-M_{B_{i}}^{(3 / 2)}(\mu, \Delta)-M_{B_{i}}^{(2)}(\mu, \Delta)+\ldots
$$

Here, $\mu$ is the renormalization scale and $\Delta$ is the quark mass independent delta-nucleon mass splitting. $M_{0}(\Delta)$ is the renormalized nucleon mass in the chiral limit, is independent of $\mu$, of $m_{q}$ and also of the $B_{i} . M_{B_{i}}^{(n)}$ is the contribution 
to the $i^{t h}$ nucleon of the order $m_{q}^{(n)} 3^{3}$ Throughout this work, we use dimensional regularization with a modified minimal subtraction $(\overline{\mathrm{MS}})$ scheme, where we have consistently subtracted terms proportional to

$$
\frac{1}{\varepsilon}-\gamma_{E}+1+\log 4 \pi
$$

The diagrams relevant to calculate the nucleon mass to NNLO are depicted in [26]. We find that the leading-order contributions to the nucleon masses are

$$
M_{B}^{(1)}=2 \alpha_{M} m_{B}+2 \sigma_{M} \operatorname{tr}\left(m_{q}\right)
$$

where

$$
m_{B}=\left\{\begin{array}{ll}
m_{u}, & B=p \\
m_{d}, & B=n
\end{array} .\right.
$$

The next-to-leading contributions are

$$
M_{B}^{(3 / 2)}=\frac{3}{16 \pi f^{2}} g_{A}^{2} m_{\pi}^{3}+\frac{8 g_{\Delta N}^{2}}{3(4 \pi f)^{2}} \mathcal{F}\left(m_{\pi}, \Delta, \mu\right),
$$

where the function $\mathcal{F}$ is defined by

$$
\begin{aligned}
\mathcal{F}(m, \delta, \mu)=\left(m^{2}-\delta^{2}\right)\left[\sqrt{\delta^{2}-m^{2}} \log \left(\frac{\delta-\sqrt{\delta^{2}-m^{2}+i \varepsilon}}{\delta+\sqrt{\delta^{2}-m^{2}+i \varepsilon}}\right)-\delta \log \left(\frac{m^{2}}{\mu^{2}}\right)\right] \\
-\frac{1}{2} \delta m^{2} \log \left(\frac{m^{2}}{\mu^{2}}\right)-\delta^{3} \log \left(\frac{4 \delta^{2}}{\mu^{2}}\right) .
\end{aligned}
$$

Finally the next-to-next-to-leading contributions to the nucleon mass are

$$
\begin{aligned}
M_{B}^{(2)}= & \left(Z_{B}-1\right) M_{B}^{(1)}+\frac{1}{4 \pi f}\left\{b_{1}^{M}\left(m_{B}\right)^{2}+b_{5}^{M} \operatorname{tr}\left(m_{q}^{2}\right)+b_{6}^{M} m_{B} \operatorname{tr}\left(m_{q}\right)+b_{8}^{M}\left[\operatorname{tr}\left(m_{q}\right)\right]^{2}\right\} \\
& -\frac{1}{(4 \pi f)^{2}} C_{\pi}^{B} \mathcal{L}\left(m_{\pi}, \mu\right)-\frac{6 \sigma_{M}}{(4 \pi f)^{2}} \operatorname{tr}\left(m_{q}\right) \mathcal{L}\left(m_{\pi}, \mu\right) \\
& +\frac{3 b^{A}}{(4 \pi f)^{3}} \overline{\mathcal{L}}\left(m_{\pi}, \mu\right)+\frac{3 b^{v A}}{4(4 \pi f)^{3}}\left[\overline{\mathcal{L}}\left(m_{\pi}, \mu\right)-\frac{1}{2} m_{\pi}^{4}\right] \\
& +\frac{1}{(4 \pi f)^{2}} \frac{27 g_{A}^{2}}{16 M_{B}}\left[\overline{\mathcal{L}}\left(m_{\pi}, \mu\right)+\frac{5}{6} m_{\pi}^{4}\right]+\frac{1}{(4 \pi f)^{2}} \frac{5 g_{\Delta N}^{2}}{2 M_{B}}\left[\overline{\mathcal{L}}\left(m_{\pi}, \mu\right)+\frac{9}{10} m_{\pi}^{4}\right] \\
& +\frac{9 g_{A}^{2} \sigma_{M}}{(4 \pi f)^{2}} \operatorname{tr}\left(m_{q}\right)\left[\mathcal{L}\left(m_{\pi}, \mu\right)+\frac{2}{3} m_{\pi}^{2}\right]+\frac{8 g_{\Delta N}^{2} \bar{\sigma}_{M}}{(4 \pi f)^{2}} \operatorname{tr}\left(m_{q}\right)\left[\mathcal{J}\left(m_{\pi}, \Delta, \mu\right)+m_{\pi}^{2}\right] \\
& +\frac{3 g_{A}^{2}}{(4 \pi f)^{2}} F_{\pi}^{B}\left[\mathcal{L}\left(m_{\pi}, \mu\right)+\frac{2}{3} m_{\pi}^{2}\right]-\frac{2 g_{\Delta N}^{2}}{(4 \pi f)^{2}} \gamma_{M} G_{\pi}^{B}\left[\mathcal{J}\left(m_{\pi}, \Delta, \mu\right)+m_{\pi}^{2}\right] .
\end{aligned}
$$

Here we have used $\left(m_{B}\right)^{2}$ which is the square of the tree-level coefficients $m_{B}$ appearing in Eq. (19). Above the wavefunction renormalization $Z_{B}$ is given by

$$
Z_{B}-1=-\frac{9 g_{A}^{2}}{2(4 \pi f)^{2}}\left[\mathcal{L}\left(m_{\pi}, \mu\right)+\frac{2}{3} m_{\pi}^{2}\right]-\frac{4 g_{\Delta N}^{2}}{(4 \pi f)^{2}}\left[\mathcal{J}\left(m_{\pi}, \Delta, \mu\right)+m_{\pi}^{2}\right] .
$$

\footnotetext{
${ }^{3}$ The renormalization scale dependence appears at each order because we are treating the LECs as polynomial functions of $\Delta$. If we instead treat this dependence explicitly and expand the nucleon mass in powers of $q$, where $\Delta \sim q$ and $m_{q} \sim q^{2}$, the mass then takes the form

$$
M_{B_{i}}=m_{0}-m_{B_{i}}^{(2)}-m_{B_{i}}^{(3)}-m_{B_{i}}^{(4)}+\ldots
$$

with $m_{B_{i}}^{(n)}$ as the renormalization scale independent mass contribution to the $i^{\text {th }}$ nucleon strictly of the order $q^{n}$. The parameter $m_{0}$ is nucleon mass in the chiral limit, but only to leading order in $\Delta$, i.e. $m_{0}=M_{0}(\Delta=0)$.
} 
TABLE I: The coefficients $C_{\pi}^{B}, F_{\pi}^{B}$, and $G_{\pi}^{B}$ in $\chi \mathrm{PT}$. Coefficients are listed for the nucleons.

\begin{tabular}{c|ccc} 
& $C_{\pi}^{B}$ & $F_{\pi}^{B}$ & $G_{\pi}^{B}$ \\
\hline$p$ & $2 \alpha_{M}\left(2 m_{u}+m_{d}\right)$ & $\alpha_{M}\left(m_{u}+2 m_{d}\right)$ & $\frac{4}{9}\left(7 m_{u}+2 m_{d}\right)$ \\
$n$ & $2 \alpha_{M}\left(m_{u}+2 m_{d}\right)$ & $\alpha_{M}\left(2 m_{u}+m_{d}\right)$ & $\frac{4}{9}\left(2 m_{u}+7 m_{d}\right)$
\end{tabular}

TABLE II: The tree-level coefficients in $\chi \mathrm{PT}$. The coefficients $m_{T},\left(m^{2}\right)_{T}$, and $\left(m m^{\prime}\right)_{T}$ are listed for the deltas $T$.

\begin{tabular}{c|ccc} 
& $m_{T}$ & $\left(m^{2}\right)_{T}$ & $\left(m^{\prime}\right)_{T}$ \\
\hline$\Delta^{++}$ & $3 m_{u}$ & $3 m_{u}^{2}$ & $3 m_{u}^{2}$ \\
$\Delta^{+}$ & $2 m_{u}+m_{d}$ & $2 m_{u}^{2}+m_{d}^{2}$ & $m_{u}^{2}+2 m_{u} m_{d}$ \\
$\Delta^{0}$ & $m_{u}+2 m_{d}$ & $m_{u}^{2}+2 m_{d}^{2}$ & $2 m_{u} m_{d}+m_{d}^{2}$ \\
$\Delta^{-}$ & $3 m_{d}$ & $3 m_{d}^{2}$ & $3 m_{d}^{2}$
\end{tabular}

The coefficients in the NNLO contribution, namely $C_{\pi}^{B}, F_{\pi}^{B}$, and $G_{\pi}^{B}$, are given in Table $\llbracket$ and depend on whether $B=p$ or $B=n$. The equations above, Eq. (22) and Eq. (23), also employ abbreviations for non-analytic functions arising from loop contributions. These functions are

$$
\begin{aligned}
\mathcal{L}(m, \mu) & =m^{2} \log \frac{m^{2}}{\mu^{2}}, \\
\overline{\mathcal{L}}(m, \mu) & =m^{4} \log \frac{m^{2}}{\mu^{2}} \\
\mathcal{J}(m, \delta, \mu) & =\left(m^{2}-2 \delta^{2}\right) \log \frac{m^{2}}{\mu^{2}}+2 \delta \sqrt{\delta^{2}-m^{2}} \log \left(\frac{\delta-\sqrt{\delta^{2}-m^{2}+i \varepsilon}}{\delta+\sqrt{\delta^{2}-m^{2}+i \varepsilon}}\right)+2 \delta^{2} \log \left(\frac{4 \delta^{2}}{\mu^{2}}\right) .
\end{aligned}
$$

The expressions we have derived in this section, as well as those throughout this work, are functions of the quark masses; e.g. $m_{\pi}$ above is merely a replacement for the combination of quark masses given in Eq. (4). These expressions, thus require the lattice practitioner to determine the quark masses. In $S U(2) \chi \mathrm{PT}$ away from the isospin limit, there are no equivalent expressions in terms of the meson masses, as one cannot independently associate $m_{u}$ and $m_{d}$ to the pion masses, which are degenerate to the order we are working. One can only equate the average value, $\frac{1}{2}\left(m_{u}+m_{d}\right)$, with the pion mass. Therefore one cannot plot the baryon masses as a function of the physical meson masses, unless one works in the isospin limit, $m_{u}=m_{d}$. This is unlike the case in the isospin limit of $S U(3)$, where there are only two independent quark masses, but three independent meson masses, and one can always convert from a quark mass expansion to a meson mass expansion via the Gell-Mann-Okubo relation. ${ }^{4}$ Lastly we remark that if one is using $\chi \mathrm{PT}$ to determine the quark masses from meson masses that are determined on the lattice, one must use the one-loop expression in $\chi \mathrm{PT}$, else one looses contributions to baryon masses that are of NNLO. Thus the quark mass expansion is the one to use in $S U(2) \chi \mathrm{PT}$, at least away from the isospin limit.

\section{Deltas}

The mass of the $i^{\text {th }}$ delta in the chiral expansion can be written as

$$
M_{T_{i}}=M_{0}(\Delta)+\Delta+M_{T_{i}}^{(1)}(\mu, \Delta)+M_{T_{i}}^{(3 / 2)}(\mu, \Delta)+M_{T_{i}}^{(2)}(\mu, \Delta)+\ldots
$$

Here, $M_{0}(\Delta)$ is the renormalized nucleon mass in the chiral limit from Eq. (17), and $\Delta$ is the renormalized nucleondelta mass splitting in the chiral limit. Both of these quantities are independent of $m_{q}$ and also of the $T_{i}$. $M_{T_{i}}^{(n)}$ is the contribution to the $i^{\text {th }}$ decuplet baryon of the order $m_{q}^{(n)}$, and $\mu$ is the renormalization scale. ${ }^{5}$

\footnotetext{
4 This problem can be avoided in $S U(4 \mid 2) \mathrm{PQ} \chi \mathrm{PT}$, as there are more independent meson masses than independent quark masses, so one can algebraically convert from the quark mass expansion to the meson mass expansion. For example, to leading order, $m_{u}=$ $\frac{f^{2}}{8 \lambda}\left(m_{\pi}^{2}-m_{j d}^{2}+m_{j u}^{2}\right)$. This would require one to know the mass of the mesons made of one valence and one sea quark. But if one is interested in the non-isospin $S U(2)$ limit of the PQ $\chi \mathrm{PT}$ expressions, the problem is unavoidable.

${ }^{5}$ As with the nucleon masses, the renormalization scale appears in each term contributing to the delta masses because we implicitly treat the LECs as polynomial functions of the mass parameter $\Delta$. If we expand out the LECs, the $\mu$-dependence disappears at each order.
} 
TABLE III: The coefficients $C_{\pi}^{T}, F_{\pi}^{T}$, and $G_{\pi}^{T}$ in $\chi \mathrm{PT}$. Coefficients are listed for the delta states $T$.

\begin{tabular}{c|ccc} 
& $C_{\pi}^{T}$ & $F_{\pi}^{T}$ & $G_{\pi}^{T}$ \\
\hline$\Delta^{++}$ & $2 m_{u}+m_{d}$ & $\frac{13}{6} m_{u}+\frac{1}{3} m_{d}$ & $\frac{4}{3} \alpha_{M} m_{u}$ \\
$\Delta^{+}$ & $\frac{5}{3} m_{u}+\frac{4}{3} m_{d}$ & $\frac{14}{9} m_{u}+\frac{17}{18} m_{d}$ & $\frac{4}{9} \alpha_{M}\left(2 m_{u}+m_{d}\right)$ \\
$\Delta^{0}$ & $\frac{4}{3} m_{u}+\frac{5}{3} m_{d}$ & $\frac{17}{18} m_{u}+\frac{14}{9} m_{d}$ & $\frac{4}{9} \alpha_{M}\left(m_{u}+2 m_{d}\right)$ \\
$\Delta^{-}$ & $m_{u}+2 m_{d}$ & $\frac{1}{3} m_{u}+\frac{13}{6} m_{d}$ & $\frac{4}{3} \alpha_{M} m_{d}$
\end{tabular}

The diagrams relevant to calculate the delta masses to NNLO are depicted in [27]. We find that the leading-order contributions to the delta masses are

$$
M_{T}^{(1)}=\frac{2}{3} \gamma_{M} m_{T}-2 \bar{\sigma}_{M} \operatorname{tr}\left(m_{q}\right)
$$

where the tree-level coefficients $m_{T}$ are given in Table $\amalg$ for the deltas $T$. The next-to-leading order contributions are

$$
M_{T}^{(3 / 2)}=-\frac{25 g_{\Delta \Delta}^{2}}{432 \pi f^{2}} m_{\pi}^{3}-\frac{2 g_{\Delta N}^{2}}{3(4 \pi f)^{2}} \mathcal{F}\left(m_{\pi},-\Delta, \mu\right)
$$

Finally the next-to-next-to-leading contributions to the delta masses are

$$
\begin{aligned}
M_{T}^{(2)}= & \left(Z_{T}-1\right) M_{T}^{(1)} \\
& +\frac{1}{4 \pi f}\left\{\frac{1}{3} t_{1}^{M}\left(m^{2}\right)_{T}+\frac{1}{3} t_{2}^{M}\left(m m^{\prime}\right)_{T}+t_{3}^{M} \operatorname{tr}\left(m_{q}^{2}\right)+\frac{1}{3} t_{4}^{M} m_{T} \operatorname{tr}\left(m_{q}\right)+t_{5}^{M}\left[\operatorname{tr}\left(m_{q}\right)\right]^{2}\right\} \\
& -\frac{2 \gamma_{M}}{(4 \pi f)^{2}} C_{\pi}^{T} \mathcal{L}\left(m_{\pi}, \mu\right)+\frac{6 \bar{\sigma}_{M}}{(4 \pi f)^{2}} \operatorname{tr}\left(m_{q}\right) \mathcal{L}\left(m_{\pi}, \mu\right)+\frac{1}{(4 \pi f)^{3}}\left(\frac{1}{2} t_{2}^{A}+3 t_{3}^{A}\right) \overline{\mathcal{L}}\left(m_{\pi}, \mu\right) \\
& +\frac{1}{4(4 \pi f)^{3}}\left[\frac{1}{2}\left(t_{2}^{\tilde{A}}+t_{2}^{v A}\right)+3\left(t_{3}^{\tilde{A}}+t_{3}^{v A}\right)\right]\left[\overline{\mathcal{L}}\left(m_{\pi}, \mu\right)-\frac{1}{2} m_{\pi}^{4}\right] \\
& -\frac{25 g_{\Delta \Delta}^{2}}{48(4 \pi f)^{2} M_{B}}\left[\overline{\mathcal{L}}\left(m_{\pi}, \mu\right)+\frac{19}{10} m_{\pi}^{4}\right]-\frac{5 g_{\Delta N}^{2}}{8(4 \pi f)^{2} M_{B}}\left[\overline{\mathcal{L}}\left(m_{\pi}, \mu\right)-\frac{1}{10} m_{\pi}^{4}\right] \\
& -\frac{25 g_{\Delta \Delta}^{2} \bar{\sigma}_{M}}{9(4 \pi f)^{2}} \operatorname{tr}\left(m_{Q}\right)\left[\mathcal{L}\left(m_{\pi}, \mu\right)+\frac{26}{15} m_{\pi}^{4}\right]-\frac{2 g_{\Delta N}^{2}}{(4 \pi f)^{2}} \operatorname{tr}\left(m_{Q}\right) \mathcal{J}\left(m_{\pi},-\Delta, \mu\right) \\
& +\frac{10 g_{\Delta \Delta}^{2} \gamma_{M}}{9(4 \pi f)^{2}} F_{\pi}^{T}\left[\mathcal{L}\left(m_{\pi}, \mu\right)+\frac{26}{15} m_{\pi}^{2}\right]-\frac{3 g_{\Delta N}^{2}}{2(4 \pi f)^{2}} G_{\pi}^{T} \mathcal{J}\left(m_{\pi},-\Delta, \mu\right) .
\end{aligned}
$$

Here we have used $\left(\mathrm{m}^{2}\right)_{T},\left(\mathrm{~mm}^{\prime}\right)_{T}$ to label the tree-level coefficients that appear in Table AlI Above the wavefunction renormalization $Z_{T}$ is given by

$$
Z_{T}-1=-\frac{25 g_{\Delta \Delta}^{2}}{18(4 \pi f)^{2}}\left[\mathcal{L}\left(m_{\pi}, \mu\right)+\frac{26}{15} m_{\pi}^{2}\right]-\frac{g_{\Delta N}^{2}}{(4 \pi f)^{2}} \mathcal{J}\left(m_{\pi},-\Delta, \mu\right) .
$$

The coefficients in the NNLO contribution, namely $C_{\pi}^{T}, F_{\pi}^{T}$, and $G_{\pi}^{T}$, are given in Table state $T$.

\section{MASS SPLITTINGS}

Having derived the nucleon and delta masses to NNLO in the chiral expansion, we now focus on the mass splittings between these states. To begin, we consider the nucleon mass splitting, which to our knowledge was first theoretically addressed in 38]. The degeneracy between the proton and neutron is broken by leading-order effects in the chiral theory, see Eq. (18). Beyond this order pion loops contribute, but to the order we are working, all the pions are degenerate, even away from the isospin limit. Thus the NLO contributions to the neutron and proton masses are the same, see Eq. (20), and the mass splitting, $M_{n}-M_{p}$, is given to NLO accuracy, entirely by the difference of the LO mass contribution in Eq. (18), and is linear in $m_{d}-m_{u}$. Any deviation from this linear mass splitting seen in lattice simulations of the nucleon masses should be a signature of the NNLO mass contributions and certain LECs that arise 
TABLE IV: The coefficient $\delta t^{M}$, which encodes the violation of the delta equal spacing rule.

\begin{tabular}{c|c}
$\delta M_{T}$ & $\delta t^{M}$ \\
\hline$M_{\Delta^{-}}-M_{\Delta^{0}}$ & $2 m_{d}$ \\
$M_{\Delta^{0}}-M_{\Delta^{+}}$ & $m_{u}+m_{d}$ \\
$M_{\Delta^{+}}-M_{\Delta^{++}}$ & $2 m_{u}$
\end{tabular}

at this order. Additionally, the nucleon mass splitting can be enhanced from that in nature by increasing the quark mass splitting on the lattice, $m_{d}-m_{u}$. This enhancement, combined with the vanishing of the NLO contribution to the mass splitting, provides us with a means of cleanly determining the NNLO nucleon mass contributions and isolating certain LECs arising at this order. These effects are normally obscured by the NLO contributions.

We find the nucleon mass splitting is given to NNLO by

$$
\begin{aligned}
M_{n}-M_{p}=-2 \alpha_{M}\left(m_{d}-m_{u}\right) & \\
+\left(m_{d}-m_{u}\right) & \left\{\frac{m_{\pi}^{2}}{(4 \pi f)^{2}}\left[8\left(g_{A}^{2} \alpha_{M}+g_{\Delta N}^{2}\left(\alpha_{M}+\frac{5}{9} \gamma_{M}\right)\right)-\left(b_{1}^{M}+b_{6}^{M}\right) \frac{\pi f^{3}}{\lambda}\right]\right. \\
& +\frac{\mathcal{L}\left(m_{\pi}, \mu\right)}{(4 \pi f)^{2}} 2 \alpha_{M}\left(6 g_{A}^{2}+1\right) \\
& \left.+\frac{\mathcal{J}\left(m_{\pi}, \Delta, \mu\right)}{(4 \pi f)^{2}} 8 g_{\Delta N}^{2}\left(\alpha_{M}+\frac{5}{9} \gamma_{M}\right)\right\} .
\end{aligned}
$$

An identical situation arises for the deltas, as their degeneracy is broken at leading order in the chiral expansion, while the next contribution to their splittings occurs at NNLO. Their mass splittings are given by $\delta M_{T}$, which stands for $M_{\Delta^{-}}-M_{\Delta^{0}}, M_{\Delta^{0}}-M_{\Delta^{+}}$and $M_{\Delta^{+}}-M_{\Delta^{++}}$. We find,

$$
\begin{aligned}
\delta M_{T}=\frac{2}{3} \gamma_{M}\left(m_{d}-m_{u}\right) & \\
+\left(m_{d}-m_{u}\right) & \left\{\frac{m_{\pi}^{2}}{(4 \pi f)^{2}}\left[\frac{\pi f^{3}}{3 \lambda}\left(t_{1}^{M}+t_{4}^{M}\right)-\frac{104}{243} g_{\Delta \Delta}^{2} \gamma_{M}\right]\right. \\
& -\frac{\mathcal{L}\left(m_{\pi}, \mu\right)}{(4 \pi f)^{2}} \frac{20 \gamma_{M}}{81}\left(\frac{27}{10}+g_{\Delta \Delta}^{2}\right) \\
& -\frac{\mathcal{J}\left(m_{\pi},-\Delta, \mu\right)}{(4 \pi f)^{2}} \frac{2 g_{\Delta N}^{2}}{3}\left(\gamma_{M}+\alpha_{M}\right) \\
& \left.+\frac{1}{12 \pi f} t_{2}^{M} \delta t^{M}\right\},
\end{aligned}
$$

where $\delta t^{M}$ is given in TableIV Considering these splittings, there are a few things to note. We know from experiment that the neutron is more massive than the proton, and we expect that this is mostly due to $m_{d}>m_{u}$. Similarly, we expect the deltas to follow a similar pattern with $\Delta^{-}$being more massive than $\Delta^{0}$, and so on, although this is presently undetermined experimentally. From these expectations and the expressions above, it is expected that $\alpha_{M}$ is negative and $\gamma_{M}$ is positive. Also, the delta masses, to a good approximation are expected to follow an equal spacing rule. In $\mathrm{HB} \chi \mathrm{PT}$, this rule is first violated at $\mathrm{NNLO}$, and even then, only by the operator in Eq. (15) with coefficient $t_{2}^{M}$. This coefficient can be isolated by taking successive differences.

Lastly we should comment that in nature isospin violation in the baryon masses has another source of the same size as the NNLO chiral effects, namely electromagnetic contributions. In lattice QCD calculations one can turn off the electric charges of the quarks. This is the scenario for which our calculations are applicable. There have been a few lattice computations of electromagnetic contributions to hadronic masses [39, 40, 41]. For a recent discussion of the electromagnetic effects in hadrons, see Ref. [42], and for a comprehensive phenomenological review, [43].

\section{SUMMARY}

The expansion parameter for heavy baryon $\chi \mathrm{PT}$ is $m_{\pi} / \Lambda_{\chi}$ as opposed to $m_{\pi}^{2} / \Lambda_{\chi}^{2}$ in the purely mesonic sector. Therefore it is crucial to calculate baryonic observables to at least NNLO to test the convergence of the heavy baryon 
chiral expansion, as well as reduce the theoretical error bars of these calculations. To this order, we are forced to introduce a large number of LECs [see Eq.(1415)], which must be determined to have any predictive power, however, this is no small feat. To NNLO, there are more than ten LECs which must be fit to determine the nucleon masses [not including those associated with the $\Delta / \Lambda_{\chi}$ expansion, see Eq. (16)], and there are only two nucleons. Some of these can be fit from observables besides the mass, but even so, not all can be reliably determined. Lattice QCD simulations provide us with another means of determining the LECs, as we have the freedom to vary the quark masses, providing a much larger data set to fit these universal constants. Today, these lattice simulations are done with quark masses which are significantly larger than those in nature. It is therefore crucial to understand the quark mass dependence of hadronic observables: in particular for nuclear physics, those of the nucleons and deltas. In this way, the LECs can be fit, allowing the lattice data to be extrapolated from the valence and sea quark masses used to their physical values, thus making rigorous $\mathrm{QCD}$ predictions of these hadronic observables.

In this work we have calculated the masses of the nucleons and the deltas in non-degenerate, two-flavor heavy baryon chiral perturbation theory to $\mathcal{O}\left(m_{q}^{2}\right)$. One can obtain from the authors a Mathematica notebook containing the derived expressions in various limits, including the partially-quenched calculations worked out in Appendix A relevant for current and future lattice data. We have highlighted the mass splittings of the nucleons and deltas as the $\mathcal{O}\left(m_{q}^{3 / 2}\right)$ contribution to these mass differences vanish, even away from the isospin limit. To LO these mass differences are proportional to $\left(m_{d}-m_{u}\right)$. Any deviations from this linear behavior are a signature of the NNLO mass contributions. This provides a clean handle to determine some of the LECs which contribute to the masses at $\mathcal{O}\left(m_{q}^{2}\right)$, which can otherwise be rather difficult to extract.

Another example of the utility of $\chi \mathrm{PT}$ is to predict the width of the deltas from the lattice. As the deltas are resonances, one cannot easily obtain their physical properties directly from real-valued Euclidean space simulations. As the lattice pion masses are brought down, one must deal with unstable particles. Instead, one can work with pion masses $m_{\pi} \gtrsim 300 \mathrm{MeV}$ for which the deltas are stable and lattice calculations can be performed more simply than the unstable case (which also has yet to be explored). In this regime, one still hopes the chiral expansion is valid, ${ }^{6}$ and uses the $\chi \mathrm{PT}$ expressions derived above, or the PQ $\chi \mathrm{PT}$ expressions derived in Appendix $\mathrm{A}$ to fit the LECs and these consequently lead to predictions for the physical delta resonances. For example, their decay widths can be found to NNLO from knowing the pion mass and the constants $f, \lambda, \Delta, \alpha_{M}, \sigma_{M}, \gamma_{M}, \bar{\sigma}_{M}$, and $g_{\Delta N}$. For simplicity the expression for the average delta width $\Im \mathrm{m}\left(\bar{M}_{T}\right)$ in terms of these parameters is

$$
\Im \mathrm{m}\left(\bar{M}_{T}\right)=\left\{\begin{array}{cc}
-\frac{g_{\Delta N}^{2}}{12 \pi f^{2}} \sqrt{\Delta^{2}-m_{\pi}^{2}}\left\{\Delta^{2}-m_{\pi}^{2}+\Delta \frac{f^{2} m_{\pi}^{2}}{24 \lambda}\left[3 \gamma_{M}+4\left(\sigma_{M}-\bar{\sigma}_{M}\right)+2 \alpha_{M}\right]\right\}, & \text { for } m_{\pi}<\Delta \\
0, & \text { for } m_{\pi}>\Delta
\end{array}\right.
$$

As a further application, the mass splitting between the nucleons and deltas can also be found from the various LECs. For the real part of the difference between the average nucleon and average delta masses, we have ${ }^{7}$

$$
\begin{aligned}
\Re \mathrm{e}\left(\bar{M}_{T}-\bar{M}_{B}\right)= & \Delta+A \operatorname{tr}\left(m_{q}\right)+B \operatorname{tr}\left(m_{q}^{2}\right)+C\left[\operatorname{tr}\left(m_{q}\right)\right]^{2} \\
& +\frac{m_{\pi}^{3}}{16 \pi f^{2}}\left[3 g_{A}^{2}-\frac{25}{27} g_{\Delta \Delta}^{2}\right]+\frac{5 g_{\Delta N}^{2}}{24 \pi^{2} f^{2}} \mathcal{F}\left(m_{\pi}, \Delta, \mu\right) \\
& +\frac{A \operatorname{tr}\left(m_{q}\right)}{(4 \pi f)^{2}}\left\{3 \mathcal{L}\left(m_{\pi}, \mu\right)-5 g_{\Delta N}^{2}\left[\mathcal{J}\left(m_{\pi}, \Delta, \mu\right)+\frac{4}{5} m_{\pi}^{2}\right]\right\} \\
& +\frac{1}{(4 \pi f)^{2}} \overline{\mathcal{L}}\left(m_{\pi}, \mu\right)\left[\frac{1}{4 \pi f} D+\frac{1}{8 M_{B}} E\right]+\frac{m_{\pi}^{4}}{(4 \pi f)^{2}}\left[\frac{1}{4 \pi f} F+\frac{1}{8 M_{B}} G\right] .
\end{aligned}
$$

Once the LECs are known from the calculation of baryon masses, say, one can use the chiral effective theory to calculate certain contributions to $\pi N \rightarrow \pi N$ or $\pi \Delta \rightarrow \pi \Delta$ scattering, for example, two processes which are complicated to explore on the lattice.

The nucleons and deltas hold an important place in hadronic physics. As the lightest spin- $\frac{1}{2}$ and spin- $\frac{3}{2}$ baryons, they have received considerable attention theoretically to compare with a wealth of experimental data. Soon lattice

\footnotetext{
6 This is only a conservative estimate as finite volume effects will modify the decay threshold of the deltas.

7 Here we have used the following replacements $A-G$ for particular combinations of the LECs: $A=\alpha_{M}+2\left(\sigma_{M}-\bar{\sigma}_{M}\right)+\gamma_{M}, B=\frac{3}{2} b_{1}^{M}+$ $b_{4}^{M}+\frac{1}{2} t_{1}^{M}+t_{3}^{M}, C=b_{5}^{M}+b_{7}^{M}+\frac{1}{3} t_{2}^{M}+\frac{1}{2} t_{4}^{M}+t_{5}^{M}, D=\frac{3}{2} b_{1}^{A}+3 b_{4}^{A}+\frac{3}{8} b_{1}^{v A}+\frac{3}{4} b_{4}^{v A}+\frac{3}{2} t_{1}^{A}+\frac{1}{2} t_{2}^{A}+3 t_{3}^{A}+\frac{3}{8}\left(t_{1}^{\tilde{A}}+t_{1}^{v A}\right)+\frac{1}{8}\left(t_{2}^{\tilde{A}}+t_{2}^{v A}\right)+\frac{3}{4}\left(t_{3}^{\tilde{A}}+t_{3}^{v A}\right)$, $E=\frac{27}{2} g_{A}^{2}+15 g_{\Delta N}^{2}-\frac{25}{6} g_{\Delta \Delta}, F=-\frac{1}{8}\left[\frac{3}{2} b_{1}^{v A}+3 b_{4}^{v A}+\frac{3}{2}\left(t_{1}^{\tilde{A}}+t_{1}^{v A}\right)+\frac{1}{2}\left(t_{2}^{\tilde{A}}+t_{2}^{v A}\right)+3\left(t_{3}^{\tilde{A}}+t_{3}^{v A}\right)\right]$, and $G=\frac{45}{4} g_{A}^{2}+\frac{37}{2} g_{\Delta N}^{2}-\frac{95}{12} g_{\Delta \Delta}^{2}$.
} 
QCD calculations will enable an understanding of the nucleon and delta observables in terms of the quark masses. A crucial first step will be determining their masses from first principles. Current and foreseeable lattice calculations will rely upon chiral extrapolations. Our expressions for the masses in $\chi \mathrm{PT}$ and PQ $\chi \mathrm{PT}$ enable such extrapolations, and provide a means to access physics that cannot be directly studied on current lattices.

\section{Acknowledgments}

We thank Martin Savage, Will Detmold and Matt Wingate for many useful discussions. This work was supported in part by the U.S. Department of Energy under Grants No. DE-FG03-97ER41014 (A.W.-L.) and DE-FG02-96ER40945 (B.C.T.).

\section{APPENDIX A: PARTIALLY QUENCHED CHIRAL PERTURBATION THEORY}

The study of PQQCD is motivated by lattice QCD simulations. Presently all lattice QCD simulations are done with quark masses that are larger than those in nature, due to the computational cost of simulating lighter quarks. The valence quarks, those connected to external fields, can be treated independently from the dynamical sea-quarks that exist only in the closed quark loops. When the masses of the valence and sea quarks are taken to be different, one has partially quenched QCD (PQQCD), and in the limit that the valence and sea quark masses are equal, one has QCD. To exclude contributions from closed valence loops, one must include ghost quarks in the effective theory of the lattice action, which are identical to the valence quarks, except that they have bosonic statistics. Thus the valence and ghost quark contributions to the PQQCD functional integral exactly cancel, leaving only the sea-quark contributions.

As in QCD, one postulates that the vacuum of PQQCD spontaneously breaks the (graded) chiral symmetry down to the vector subgroup, and that one can perturb about the massless quark limit. The emerging low-energy effective theory of PQQCD is partially quenched $\chi \mathrm{PT}(\mathrm{PQ} \chi \mathrm{PT})$ 44, 45, 46, 47, 48, 49]. Since PQQCD retains an axial anomaly, the flavor singlet field can be integrated out and there are no additional operators required that involve the singlet field. Moreover because the sea sector of PQQCD contains QCD, the same LECs of $\chi \mathrm{PT}$ appear in PQ $\chi \mathrm{PT}$ with the same numerical values. Hence there is a means to determine $\chi \mathrm{PT}$ parameters using PQQCD lattice simulations. This hope has generated much activity in calculating baryon masses in partially quenched theories [26, 27, 50, 51, 52].

In PQQCD, the quark part of the Lagrangian is

$$
\mathfrak{L}=\sum_{j, k=1}^{6} \bar{Q}^{j}\left(i \not D-m_{Q}\right)_{j}^{k} Q_{k} .
$$

This differs from the usual $S U(2)$ Lagrangian of QCD by the inclusion of four extra quarks; two bosonic ghost quarks, $(\tilde{u}, \tilde{d})$, and two fermionic sea quarks, $(j, l)$, in addition to the light physical quarks $(u, d)$. The six quark fields transform in the fundamental representation of the graded $S U(4 \mid 2)$ group. They have been accommodated in the six-component vector

$$
Q^{T}=(u, d, j, l, \tilde{u}, \tilde{d}) .
$$

The quark fields obey the graded equal-time commutation relation

$$
Q_{i}^{\alpha}(\mathbf{x}) Q_{j}^{\beta \dagger}(\mathbf{y})-(-1)^{\eta_{i} \eta_{j}} Q_{j}^{\beta \dagger}(\mathbf{y}) Q_{i}^{\alpha}(\mathbf{x})=\delta^{\alpha \beta} \delta_{i j} \delta^{3}(\mathbf{x}-\mathbf{y}),
$$

where $\alpha, \beta$ and $i, j$ are spin and flavor indices, respectively. Analogous graded equal-time commutation relations can be written for two $Q$ 's and two $Q^{\dagger}$ 's. The grading factors

$$
\eta_{k}=\left\{\begin{array}{l}
1 \text { for } k=1,2,3,4 \\
0 \text { for } k=5,6
\end{array},\right.
$$

take into account the different statistics of the quark fields of PQQCD. The quark mass matrix of $S U(4 \mid 2)$ is given by

$$
m_{Q}=\operatorname{diag}\left(m_{u}, m_{d}, m_{j}, m_{l}, m_{u}, m_{d}\right) .
$$

QCD is recovered in the limit $m_{j} \rightarrow m_{u}$ and $m_{l} \rightarrow m_{d}$. 


\section{Partially Quenched $\chi \mathbf{P T}$ Lagrangian}

For massless quarks, the theory corresponding to the Lagrangian in Eq. (A1) has a graded $S U(4 \mid 2)_{L} \otimes$ $S U(4 \mid 2)_{R} \otimes U(1)_{V}$ symmetry which is assumed to be spontaneously broken down to $S U(4 \mid 2)_{V} \otimes U(1)_{V}$ in analogy with QCD. The effective low-energy theory obtained by perturbing about the physical vacuum state of PQQCD is $\mathrm{PQ} \chi \mathrm{PT}$ [4, 45, 46, 47, 48, 49]. The emerging pseudo-Goldstone mesons have dynamics described at leading order in the chiral expansion by the Lagrangian

$$
\mathfrak{L}=\frac{f^{2}}{8} \operatorname{str}\left(\partial^{\mu} \Sigma^{\dagger} \partial_{\mu} \Sigma\right)+\lambda \operatorname{str}\left(m_{Q} \Sigma^{\dagger}+m_{Q}^{\dagger} \Sigma\right)
$$

where

$$
\Sigma=\exp \left(\frac{2 i \Phi}{f}\right)=\xi^{2}
$$

and the meson fields appear in

$$
\Phi=\left(\begin{array}{cc}
M & \chi^{\dagger} \\
\chi & \tilde{M}
\end{array}\right)
$$

The operation $\operatorname{str}()$ in Eq. (A6) is a graded flavor trace. $M$ and $\tilde{M}$ are matrices containing bosonic mesons (with quantum numbers of $q \bar{q}$ pairs and $\tilde{q} \overline{\tilde{q}}$ pairs, respectively), while $\chi$ and $\chi^{\dagger}$ are matrices containing fermionic mesons (with quantum numbers of $\tilde{q} \bar{q}$ pairs and $q \overline{\tilde{q}}$ pairs, respectively). The upper $2 \times 2$ block of $M$ contains the familiar pions and the remaining components are mesons formed with one or two sea quarks, see e.g. 51]. Expanding the Lagrangian Eq. (A6) to leading order, one finds that mesons with quark content $Q Q^{\prime}$ are canonically normalized with their masses given by

$$
m_{Q Q^{\prime}}^{2}=\frac{4 \lambda}{f^{2}}\left(m_{Q}+m_{Q^{\prime}}\right)
$$

The flavor singlet field is defined to be $\Phi_{0}=\operatorname{str}(\Phi) / \sqrt{2}$. As PQQCD has a strong axial anomaly $U(1)_{A}$, the mass of the singlet field is on the order of the chiral symmetry breaking scale and has been integrated out of the theory [48]. In this limit, however, the $\eta$ two-point correlation functions deviate from their form in $\chi \mathrm{PT}$. For $a, b=u, d, j, l, \tilde{u}, \tilde{d}$, the leading-order $\eta_{a} \eta_{b}$ propagator with non-degenerate sea-quarks is

$$
\mathcal{G}_{\eta_{a} \eta_{b}}=\frac{i \epsilon_{a} \delta_{a b}}{q^{2}-m_{\eta_{a}}^{2}+i \epsilon}-\frac{i}{2} \frac{\epsilon_{a} \epsilon_{b}\left(q^{2}-m_{j j}^{2}\right)\left(q^{2}-m_{l l}^{2}\right)}{\left(q^{2}-m_{\eta_{a}}^{2}+i \epsilon\right)\left(q^{2}-m_{\eta_{b}}^{2}+i \epsilon\right)\left(q^{2}-m_{X}^{2}+i \epsilon\right)},
$$

where

$$
\epsilon_{a}=(-1)^{1+\eta_{a}}
$$

The mass $m_{x y}$ is the mass of a meson composed of (anti)-quarks of flavor $x$ and $y$, while the mass $m_{X}$ is defined as $m_{X}^{2}=\frac{1}{2}\left(m_{j j}^{2}+m_{l l}^{2}\right)$. The flavor neutral propagator can be conveniently rewritten as

$$
\mathcal{G}_{\eta_{a} \eta_{b}}=\epsilon_{a} \delta_{a b} P_{a}+\epsilon_{a} \epsilon_{b} \mathcal{H}_{a b}\left(P_{a}, P_{b}, P_{X}\right)
$$

where

$$
\begin{gathered}
P_{a}=\frac{i}{q^{2}-m_{\eta_{a}}^{2}+i \epsilon}, P_{b}=\frac{i}{q^{2}-m_{\eta_{b}}^{2}+i \epsilon}, P_{X}=\frac{i}{q^{2}-m_{X}^{2}+i \epsilon}, \\
\mathcal{H}_{a b}(A, B, C)=-\frac{1}{2}\left[\frac{\left(m_{j j}^{2}-m_{\eta_{a}}^{2}\right)\left(m_{l l}^{2}-m_{\eta_{a}}^{2}\right)}{\left(m_{\eta_{a}}^{2}-m_{\eta_{b}}^{2}\right)\left(m_{\eta_{a}}^{2}-m_{X}^{2}\right)} A-\frac{\left(m_{j j}^{2}-m_{\eta_{b}}^{2}\right)\left(m_{l l}^{2}-m_{\eta_{b}}^{2}\right)}{\left(m_{\eta_{a}}^{2}-m_{\eta_{b}}^{2}\right)\left(m_{\eta_{b}}^{2}-m_{X}^{2}\right)} B\right. \\
\left.+\frac{\left(m_{X}^{2}-m_{j j}^{2}\right)\left(m_{X}^{2}-m_{l l}^{2}\right)}{\left(m_{X}^{2}-m_{\eta_{a}}^{2}\right)\left(m_{X}^{2}-m_{\eta_{b}}^{2}\right)} C\right] .
\end{gathered}
$$


For our calculation, the nucleons and deltas must also be included in PQ $\chi \mathrm{PT}$ [50, 51]. A straightforward way to do this is to use interpolating fields that have non-zero overlap with the nucleons and deltas. This leads to the tensor $\mathcal{B}^{i j k}$, which describes a 70-dimensional representation of SU(4|2), and the tensor $\mathcal{T}^{i j k}$, which describes a 44-dimensional representation of $\mathrm{SU}(4 \mid 2)$, see [51]. The nucleon doublet $N_{i}$ is embedded in $\mathcal{B}_{i j k}$ as

$$
\mathcal{B}_{i j k}=\frac{1}{\sqrt{6}}\left(\varepsilon_{i j} N_{k}+\varepsilon_{i k} N_{j}\right),
$$

where the indices $i, j$, and $k$ are restricted to 1 or 2 . The deltas are contained simply as $\mathcal{T}^{i j k}=T^{i j k}$ for $i, j$, and $k$ restricted to 1 or 2 .

To write down the PQ $\chi \mathrm{PT}$ Lagrangian in the baryon sector, we must also include the appropriate grading factors in the contraction of flavor indices. Such factors are included in the () notation defined in [51, 53]. The free Lagrangian is given by

$$
\begin{aligned}
\mathfrak{L}= & (\overline{\mathcal{B}} i v \cdot D \mathcal{B})+2 \alpha_{M}^{(P Q)}(\overline{\mathcal{B}} \mathcal{B} \mathcal{M})+2 \beta_{M}^{(P Q)}(\overline{\mathcal{B}} \mathcal{M B})+2 \sigma_{M}^{(P Q)}(\overline{\mathcal{B}} \mathcal{B}) \operatorname{str}(\mathcal{M}) \\
& -\left(\overline{\mathcal{T}}^{\mu}[i v \cdot D-\Delta] \mathcal{T}_{\mu}\right)+2 \gamma_{M}^{(P Q)}\left(\overline{\mathcal{T}}^{\mu} \mathcal{M} \mathcal{T}_{\mu}\right)-2 \bar{\sigma}_{M}^{(P Q)}\left(\overline{\mathcal{T}}^{\mu} \mathcal{T}_{\mu}\right) \operatorname{str}(\mathcal{M})
\end{aligned}
$$

Above, the covariant derivative's action on either the $\mathcal{B}$ or the $\mathcal{T}$ fields is defined by

$$
\left(D^{\mu} \mathcal{B}\right)_{i j k}=\partial^{\mu} \mathcal{B}_{i j k}+\left(V^{\mu}\right)_{i}^{i^{\prime}} \mathcal{B}_{i^{\prime} j k}+(-)^{\eta_{i}\left(\eta_{j}+\eta_{j^{\prime}}\right)}\left(V^{\mu}\right)_{j}^{j^{\prime}} \mathcal{B}_{i j^{\prime} k}+(-)^{\left(\eta_{i}+\eta_{j}\right)\left(\eta_{k}+\eta_{k^{\prime}}\right)}\left(V^{\mu}\right)_{k}{ }^{k^{\prime}} \mathcal{B}_{i j k^{\prime}}
$$

and the operators $A^{\mu}, V^{\mu}$ and $\mathcal{M}$ are all defined analogously to those in $\chi \mathrm{PT}$. The free Lagrangian above contains more operators than the corresponding $\mathrm{SU}(2)$ Lagrangian. For this reason we have appended $(P Q)$ superscripts to the coefficients. The relations between the free Lagrangian parameters of $\mathrm{PQ} \chi \mathrm{PT}$ and $\chi \mathrm{PT}$ are found by matching. One finds

$$
\begin{aligned}
\alpha_{M} & =\frac{2}{3} \alpha_{M}^{(P Q)}-\frac{1}{3} \beta_{M}^{(P Q)} \\
\sigma_{M} & =\sigma_{M}^{(P Q)}+\frac{1}{6} \alpha_{M}^{(P Q)}+\frac{2}{3} \beta_{M}^{(P Q)} \\
\gamma_{M} & =\gamma_{M}^{(P Q)} \\
\bar{\sigma}_{M} & =\bar{\sigma}_{M}^{(P Q)} .
\end{aligned}
$$

The Lagrangian describing the interactions of the $\mathbf{7 0}$ and $\mathbf{4 4}$ baryons with the pseudo-Goldstone particles is

$$
\begin{aligned}
\mathfrak{L} & =2 \alpha\left(\overline{\mathcal{B}} S^{\mu} \mathcal{B} A_{\mu}\right)+2 \beta\left(\overline{\mathcal{B}} S^{\mu} A_{\mu} \mathcal{B}\right)+2 \mathcal{H}\left(\overline{\mathcal{T}}^{\nu} S^{\mu} A_{\mu} \mathcal{T}_{\nu}\right) \\
& +\sqrt{\frac{3}{2}} \mathcal{C}\left[\left(\overline{\mathcal{T}}^{\nu} A_{\nu} \mathcal{B}\right)+\left(\overline{\mathcal{B}} A_{\nu} \mathcal{T}^{\nu}\right)\right]
\end{aligned}
$$

Matching this Lagrangian onto the $\mathrm{SU}(2)$ chiral Lagrangian in Eq. (12), we find $\mathcal{H}=g_{\Delta \Delta}$ and $\mathcal{C}=-g_{\Delta N}$. Furthermore, we follow [51] and choose to express $\alpha$ and $\beta$ in terms of new parameters $g_{A}$ and $g_{1}$, namely

$$
\begin{aligned}
& \alpha=\frac{4}{3} g_{A}+\frac{1}{3} g_{1}, \\
& \beta=\frac{2}{3} g_{1}-\frac{1}{3} g_{A},
\end{aligned}
$$

so that $g_{A}$ has the same value as in $\chi \mathrm{PT}$.

At higher orders in the chiral expansion, the situation in PQ $\chi \mathrm{PT}$ parallels that of $\chi \mathrm{PT}$ in Sec. II Recall that at higher orders, the Lagrangian can contain arbitrary functions of $\Delta / \Lambda_{\chi}$. We take this into account by implicitly treating the leading-order coefficients as functions of $\Delta / \Lambda_{\chi}$ expanded out to the required order. To maintain the Lorentz invariance of the theory, we use reparameterization invariance to generate the higher dimensional operators with fixed coefficients. In PQ $\chi \mathrm{PT}$ the fixed coefficient Lagrangian is given by

$$
\begin{aligned}
\mathfrak{L}= & -\left(\overline{\mathcal{B}} \frac{D_{\perp}^{2}}{2 M_{B}} \mathcal{B}\right)+\alpha\left[\left(\overline{\mathcal{B}} \frac{i \overleftarrow{D} \cdot S}{M_{B}} \mathcal{B} v \cdot A\right)-\left(\overline{\mathcal{B}} \frac{S \cdot i \vec{D}}{M_{B}} \mathcal{B} v \cdot A\right)\right] \\
& +\beta\left[\left(\overline{\mathcal{B}} \frac{i \overleftarrow{D} \cdot S}{M_{B}} v \cdot A \mathcal{B}\right)-\left(\overline{\mathcal{B}} v \cdot A \frac{S \cdot i \vec{D}}{M_{B}} \mathcal{B}\right)\right]+\left(\overline{\mathcal{T}}^{\mu} \frac{D_{\perp}^{2}}{2 M_{B}} \mathcal{T}_{\mu}\right)
\end{aligned}
$$




$$
+\mathcal{H}\left[\left(\overline{\mathcal{T}}^{\mu} \frac{i \overleftarrow{D} \cdot S}{M_{B}} v \cdot A \mathcal{T}_{\mu}\right)-\left(\overline{\mathcal{T}}^{\mu} v \cdot A \frac{S \cdot i \vec{D}}{M_{B}} \mathcal{T}_{\mu}\right)\right]
$$

As far as operators with unfixed coefficients, for the nucleon mass we have the following partially quenched operators with two insertions of the axial-vector pion fields

$$
\begin{aligned}
& \mathfrak{L}=\frac{1}{(4 \pi f)}\left\{b_{1}^{A(P Q)} \overline{\mathcal{B}}^{k j i}(A \cdot A)_{i}{ }^{n} \mathcal{B}_{n j k}+b_{2}^{A(P Q)}(-)^{\left(\eta_{i}+\eta_{j}\right)\left(\eta_{k}+\eta_{n}\right)} \overline{\mathcal{B}}^{k j i}(A \cdot A)_{k}{ }^{n} \mathcal{B}_{i j n}\right. \\
& +b_{3}^{A(P Q)}(-)^{\eta_{l}\left(\eta_{j}+\eta_{n}\right)} \overline{\mathcal{B}}^{k j i}\left(A_{\mu}\right)_{i}{ }^{l}\left(A^{\mu}\right)_{j}{ }_{j} \mathcal{B}_{l n k}+b_{4}^{A(P Q)}(-)^{\eta_{j} \eta_{n}+1} \overline{\mathcal{B}}^{k j i}\left(A_{\mu}\right)_{i}{ }^{n}\left(A^{\mu}\right)_{j}{ }_{j} \mathcal{B}_{l n k} \\
& +b_{5}^{A(P Q)} \overline{\mathcal{B}}^{k j i} \mathcal{B}_{i j k} \operatorname{Tr}(A \cdot A)+b_{1}^{v A(P Q)} \overline{\mathcal{B}}^{k j i}(v \cdot A v \cdot A)_{i}{ }^{n} \mathcal{B}_{n j k} \\
& +b_{2}^{v A(P Q)}(-)^{\left(\eta_{i}+\eta_{j}\right)\left(\eta_{k}+\eta_{n}\right)} \overline{\mathcal{B}}^{k j i}(v \cdot A v \cdot A)_{k}{ }^{n} \mathcal{B}_{i j n} \\
& +b_{3}^{v A(P Q)}(-)^{\eta_{l}\left(\eta_{j}+\eta_{n}\right)} \overline{\mathcal{B}}^{k j i}(v \cdot A)_{i}^{l}(v \cdot A)_{j}{ }^{n} \mathcal{B}_{\text {lnk }} \\
& \left.+b_{4}^{v A(P Q)}(-)^{\eta_{j} \eta_{n}+1} \overline{\mathcal{B}}^{k j i}(v \cdot A)_{i}{ }^{n}(v \cdot A)_{j}{ }^{l} \mathcal{B}_{l n k}+b_{5}^{v A(P Q)} \overline{\mathcal{B}}^{k j i} \mathcal{B}_{i j k} \operatorname{Tr}(v \cdot A v \cdot A)\right\},
\end{aligned}
$$

and the following operators with two insertions of the mass operator

$$
\begin{aligned}
\mathfrak{L}= & \frac{1}{(4 \pi f)}\left\{b_{1}^{M(P Q)} \overline{\mathcal{B}}^{k j i}(\mathcal{M M})_{i}{ }^{n} \mathcal{B}_{n j k}+b_{2}^{M(P Q)}(-)^{\left(\eta_{i}+\eta_{j}\right)\left(\eta_{k}+\eta_{n}\right)} \overline{\mathcal{B}}^{k j i}(\mathcal{M M})_{k}{ }^{n} \mathcal{B}_{i j n}\right. \\
& +b_{3}^{M(P Q)}(-)^{\eta_{l}\left(\eta_{j}+\eta_{n}\right)} \overline{\mathcal{B}}^{k j i}(\mathcal{M})_{i}{ }^{l}(\mathcal{M})_{j}{ }^{n} \mathcal{B}_{l n k}+b_{4}^{M(P Q)}(-)^{\eta_{j} \eta_{n}+1} \overline{\mathcal{B}}^{k j i}(\mathcal{M})_{i}{ }^{n}(\mathcal{M})_{j}{ }_{j} \mathcal{B}_{l n k} \\
& +b_{5}^{M(P Q)} \overline{\mathcal{B}}^{k j i} \mathcal{B}_{i j k} \operatorname{str}(\mathcal{M M})+b_{6}^{M(P Q)} \overline{\mathcal{B}}^{k j i}(\mathcal{M})_{i}{ }^{n} \mathcal{B}_{n j k} \operatorname{str}(\mathcal{M}) \\
& \left.+b_{7}^{M(P Q)}(-)^{\left(\eta_{i}+\eta_{j}\right)\left(\eta_{k}+\eta_{n}\right)} \overline{\mathcal{B}}^{k j i}(\mathcal{M})_{k}{ }^{n} \mathcal{B}_{i j n} \operatorname{str}(\mathcal{M})+b_{8}^{M(P Q)} \overline{\mathcal{B}}^{k j i} \mathcal{B}_{i j k}[\operatorname{str}(\mathcal{M})]^{2}\right\}
\end{aligned}
$$

Matching the operators with coefficients $b_{j}^{M(P Q)}$ above onto the SU(2) Lagrangian in Eq. (14), we find

$$
\begin{aligned}
b_{1}^{M} & =-\frac{1}{3} b_{1}^{M(P Q)}+\frac{2}{3} b_{2}^{M(P Q)}-\frac{1}{3} b_{3}^{M(P Q)}+\frac{1}{2} b_{4}^{M(P Q)} \\
b_{5}^{M} & =\frac{2}{3} b_{1}^{M(P Q)}+\frac{1}{6} b_{2}^{M(P Q)}-\frac{1}{6} b_{3}^{M(P Q)}+\frac{1}{6} b_{4}^{M(P Q)}+b_{5}^{M(P Q)} \\
b_{6}^{M} & =\frac{1}{2} b_{3}^{M(P Q)}-\frac{1}{3} b_{4}^{M(P Q)}-\frac{1}{3} b_{6}^{M(P Q)}+\frac{2}{3} b_{7}^{M(P Q)} \\
b_{8}^{M} & =\frac{1}{6} b_{3}^{M(P Q)}-\frac{1}{6} b_{4}^{M(P Q)}+\frac{2}{3} b_{6}^{M(P Q)}+\frac{1}{6} b_{7}^{M(P Q)}+b_{8}^{M(P Q)}
\end{aligned}
$$

Carrying out the matching for the operators with coefficients $b_{j}^{A(P Q)}$ and $b_{j}^{v A(P Q)}$ leads to the relations

$$
b^{A, v A}=\frac{1}{2} b_{1}^{A, v A(P Q)}+\frac{1}{2} b_{2}^{A, v A(P Q)}-\frac{1}{3} b_{3}^{A, v A(P Q)}+\frac{5}{12} b_{4}^{A, v A(P Q)}+b_{5}^{A, v A(P Q)} .
$$

Higher-dimensional operators that contribute to the masses of the deltas in PQ $\chi \mathrm{PT}$ arise similarly from two insertions of the axial-vector pion fields

$$
\begin{aligned}
\mathfrak{L}= & \frac{1}{4 \pi f}\left\{t_{1}^{A(P Q)} \overline{\mathcal{T}}_{\mu}^{k j i}\left(A_{\nu} A^{\nu}\right)_{i}^{i^{\prime}} \mathcal{T}_{i^{\prime} j k}^{\mu}+t_{2}^{A}(-)^{\eta_{i^{\prime}}\left(\eta_{j}+\eta_{j^{\prime}}\right)} \overline{\mathcal{T}}_{\mu}^{k j i}\left(A_{\nu}\right)_{i}^{i^{\prime}}\left(A^{\nu}\right)_{j}^{j^{\prime}} \mathcal{T}_{i^{\prime} j^{\prime} k}^{\mu}\right. \\
& +t_{3}^{A(P Q)}\left(\overline{\mathcal{T}}_{\mu} \mathcal{T}^{\mu}\right) \operatorname{str}\left(A_{\nu} A^{\nu}\right)+t_{1}^{\tilde{A}(P Q)} \overline{\mathcal{T}}_{\mu}^{k j i}\left(A^{\mu} A_{\nu}\right)_{i}^{i^{\prime}} \mathcal{T}_{i^{\prime} j k}^{\nu}+t_{2}^{\tilde{A}} \overline{\mathcal{T}}_{\mu}^{k j i}\left(A^{\mu}\right)_{i}^{i^{\prime}}\left(A_{\nu}\right)_{j}^{j^{\prime}} \mathcal{T}_{i^{\prime} j^{\prime} k}^{\nu} \\
& +t_{3}^{\tilde{A}(P Q)}\left(\overline{\mathcal{T}}_{\mu} \mathcal{T}^{\nu}\right) \operatorname{str}\left(A^{\mu} A_{\nu}\right)+t_{1}^{v A(P Q)} \overline{\mathcal{T}}_{\mu}^{k j i}(v \cdot A v \cdot A)_{i}^{i^{\prime}} \mathcal{T}_{i^{\prime} j k}^{\mu}+t_{2}^{v A} \overline{\mathcal{T}}_{\mu}^{k j i}(v \cdot A)_{i}^{i^{\prime}}(v \cdot A)_{j}^{j^{\prime}} \mathcal{T}_{i^{\prime} j^{\prime} k}^{\mu} \\
& \left.+t_{3}^{v A(P Q)}\left(\overline{\mathcal{T}}_{\mu} \mathcal{T}^{\mu}\right) \operatorname{str}(v \cdot A v \cdot A)\right\}
\end{aligned}
$$


and two insertions of the mass operator

$$
\begin{aligned}
\mathfrak{L}= & \frac{1}{4 \pi f}\left\{t_{1}^{M} \overline{\mathcal{T}}_{\mu}^{k j i}(\mathcal{M M})_{i}{ }^{i^{\prime}} \mathcal{T}_{i^{\prime} j k}^{\mu}+t_{2}^{M}(-)^{\eta_{i^{\prime}}\left(\eta_{j}+\eta_{j^{\prime}}\right)} \overline{\mathcal{T}}_{\mu}^{k j i}(\mathcal{M})_{i}{ }^{i^{\prime}}(\mathcal{M})_{j}{ }^{j^{\prime}} \mathcal{T}_{i^{\prime} j^{\prime} k}^{\mu}\right. \\
& \left.+t_{3}^{M}\left(\overline{\mathcal{T}}_{\mu} \mathcal{T}^{\mu}\right) \operatorname{str}(\mathcal{M} \mathcal{M})+t_{4}^{M}\left(\overline{\mathcal{T}}_{\mu} \mathcal{M} \mathcal{T}^{\mu}\right) \operatorname{str}(\mathcal{M})+t_{5}^{M}\left(\overline{\mathcal{T}}_{\mu} \mathcal{T}^{\mu}\right)[\operatorname{str}(\mathcal{M} \mathcal{M})]^{2}\right\} .
\end{aligned}
$$

The coefficients of these operators which do not have the superscript, $(P Q), t_{2}^{A}, t_{2}^{\tilde{A}}, t_{2}^{v A}$, and $t_{i}^{M}$ have the same numerical values as those in the $\mathrm{SU}(2)$ Lagrangian, Eq. (15). The operators with the $(P Q)$ superscript are related to the $S U(2)$ LECs by the following relations,

$$
t_{3}^{A, v A, \tilde{A}}=\frac{1}{2} t_{1}^{A, v A, \tilde{A}(P Q)}+t_{3}^{A, v A, \tilde{A}(P Q)}
$$

\section{Nucleons}

The relevant diagrams needed to obtain the nucleon mass to NNLO in PQ $\chi \mathrm{PT}$ are depicted in [26] and include hairpin contributions from the flavor-diagonal states. To leading order in $\mathrm{PQ} \chi \mathrm{PT}$ the nucleon masses are

$$
M_{B}^{(1)}=\frac{1}{3} \alpha_{M}^{(P Q)} m_{B}^{\prime \prime}+\frac{1}{3} \beta_{M}^{(P Q)} m_{B}^{\prime}+2 \sigma_{M}^{(P Q)} \operatorname{str}\left(m_{Q}\right)
$$

where the tree-level coefficients $m_{B}^{\prime}$ and $m_{B}^{\prime \prime}$ depend on the valence quark masses and are given in Table $\nabla$ next-to-leading contributions to the nucleon masses take the form

$$
\begin{aligned}
M_{B}^{(3 / 2)}= & \frac{1}{8 \pi f^{2}}\left[\sum_{\phi} A_{\phi}^{B} m_{\phi}^{3}+\sum_{\phi \phi^{\prime}} A_{\phi \phi^{\prime}}^{B} \mathcal{M}^{3}\left(m_{\phi}, m_{\phi^{\prime}}\right)\right] \\
& +\frac{2 g_{\Delta N}^{2}}{(4 \pi f)^{2}}\left[\sum_{\phi} B_{\phi}^{B} \mathcal{F}\left(m_{\phi}, \Delta, \mu\right)+\sum_{\phi \phi^{\prime}} B_{\phi \phi^{\prime}}^{B} \mathcal{F}\left(m_{\phi}, m_{\phi^{\prime}}, \Delta, \mu\right)\right],
\end{aligned}
$$

where $\mathcal{F}(m, \delta, \mu)$ is given in Eq. (21). Additionally we have employed the abbreviations

$$
\begin{aligned}
\mathcal{M}^{n}\left(m_{\phi}, m_{\phi^{\prime}}\right) & =\mathcal{H}_{\phi \phi^{\prime}}\left(m_{\phi}^{n}, m_{\phi^{\prime}}^{n}, m_{X}^{n}\right), \\
\mathcal{F}\left(m_{\phi}, m_{\phi^{\prime}}, \delta, \mu\right) & =\mathcal{H}_{\phi \phi^{\prime}}\left[\mathcal{F}\left(m_{\phi}, \delta, \mu\right), \mathcal{F}\left(m_{\phi^{\prime}}, \delta, \mu\right), \mathcal{F}\left(m_{X}, \delta, \mu\right)\right],
\end{aligned}
$$

for contributions arising from the hairpin diagrams. The sums involving these two functions are over pairs of flavorneutral states in the quark basis, e.g., above $\phi \phi^{\prime}$ runs over $\eta_{u} \eta_{u}, \eta_{u} \eta_{d}$, and $\eta_{d} \eta_{d}$. In this way there is no double counting. The remaining sums in Eq. A36 are over all loop mesons $\phi$ of mass $m_{\phi}$. The coefficients $A_{\phi}^{B}, A_{\phi \phi^{\prime}}^{B}, B_{\phi}^{B}$ and $B_{\phi \phi^{\prime}}^{B}$ appear in Table VI

Finally the next-to-next-to-leading contributions to the nucleon mass are

$$
\begin{aligned}
M_{B}^{(2)}= & \left(Z_{B}-1\right) M_{B}^{(1)}+\frac{1}{4 \pi f}\left[\frac{1}{3} b_{1}^{M(P Q)}\left(m^{2}\right)_{B}+\frac{1}{6} b_{2}^{M(P Q)}\left(m^{2}\right)_{B}^{\prime}+\frac{1}{6} b_{3}^{M(P Q)}\left(m m^{\prime}\right)_{B}+\frac{1}{6} b_{4}^{M(P Q)}\left(m m^{\prime}\right)_{B}^{\prime}\right. \\
& \left.+b_{5}^{M(P Q)} \operatorname{str}\left(m_{Q}^{2}\right)+\frac{1}{3} b_{6}^{M(P Q)} m_{B}^{\prime} \operatorname{str}\left(m_{Q}\right)+\frac{1}{6} b_{7}^{M(P Q)} m_{B}^{\prime \prime} \operatorname{str}\left(m_{Q}\right)+b_{8}^{M(P Q)}\left[\operatorname{str}\left(m_{Q}\right)\right]^{2}\right] \\
& -\frac{1}{(4 \pi f)^{2}}\left[\sum_{\phi} C_{\phi}^{B} \mathcal{L}\left(m_{\phi}, \mu\right)+\sum_{\phi \phi^{\prime}} C_{\phi, \phi^{\prime}}^{B} \mathcal{L}\left(m_{\phi}, m_{\phi^{\prime}}, \mu\right)\right] \\
& -\frac{2 \sigma_{M}^{(P Q)}}{(4 \pi f)^{2}}\left[\sum_{\phi} \bar{C}_{\phi} \mathcal{L}\left(m_{\phi}, \mu\right)+\sum_{\phi \phi^{\prime}} \bar{C}_{\phi \phi^{\prime}} \mathcal{L}\left(m_{\phi}, m_{\phi^{\prime}}, \mu\right)\right] \\
& +\frac{1}{(4 \pi f)^{3}} \sum_{\phi}\left(b_{1}^{A(P Q)} D_{\beta, \phi}^{B}+b_{2}^{A(P Q)} D_{\alpha, \phi}^{B}+b_{3}^{A(P Q)} E_{\phi}^{B}+b_{4}^{A(P Q)} E_{\phi}^{\prime B}+b_{5}^{A(P Q)} \bar{D}_{\phi}\right) \overline{\mathcal{L}}\left(m_{\phi}, \mu\right)
\end{aligned}
$$




$$
\begin{aligned}
& +\frac{1}{(4 \pi f)^{3}} \sum_{\phi \phi^{\prime}}\left(b_{1}^{A(P Q)} D_{\beta, \phi \phi^{\prime}}^{B}+b_{2}^{A(P Q)} D_{\alpha, \phi \phi^{\prime}}^{B}+b_{3}^{A(P Q)} E_{\phi \phi^{\prime}}^{B}+b_{4}^{A(P Q)} E_{\phi \phi^{\prime}}^{\prime B}+b_{5}^{A(P Q)} \bar{D}_{\phi \phi^{\prime}}\right) \overline{\mathcal{L}}\left(m_{\phi}, m_{\phi^{\prime}}, \mu\right) \\
& +\frac{1}{4(4 \pi f)^{3}} \sum_{\phi}\left(b_{1}^{v A(P Q)} D_{\beta, \phi}^{B}+b_{2}^{v A(P Q)} D_{\alpha, \phi}^{B}+b_{3}^{v A(P Q)} E_{\phi}^{B}+b_{4}^{v A(P Q)} E_{\phi}^{\prime B}+b_{5}^{v A(P Q)} \bar{D}_{\phi}\right) \\
& \quad \times\left[\overline{\mathcal{L}}\left(m_{\phi}, \mu\right)-\frac{1}{2} m_{\phi}^{4}\right] \\
& +\frac{1}{4(4 \pi f)^{3}} \sum_{\phi \phi^{\prime}}\left(b_{1}^{v A(P Q)} D_{\beta, \phi \phi^{\prime}}^{B}+b_{2}^{v A(P Q)} D_{\alpha, \phi \phi^{\prime}}^{B}+b_{3}^{v A(P Q)} E_{\phi \phi^{\prime}}^{B}+b_{4}^{v A(P Q)} E_{\phi \phi^{\prime}}^{\prime B}+b_{5}^{v A(P Q)} \bar{D}_{\phi \phi^{\prime}}\right) \\
& \quad \times\left[\overline{\mathcal{L}}\left(m_{\phi}, m_{\phi^{\prime}}, \mu\right)-\frac{1}{2} \mathcal{M}^{4}\left(m_{\phi}, m_{\phi^{\prime}}\right)\right] \\
& +\frac{9}{8 M_{B}} \frac{1}{(4 \pi f)^{2}}\left\{\sum_{\phi} A_{\phi}^{B}\left[\overline{\mathcal{L}}\left(m_{\phi}, \mu\right)+\frac{5}{6} m_{\phi}^{4}\right]+\sum_{\phi \phi^{\prime}} A_{\phi \phi^{\prime}}^{B}\left[\overline{\mathcal{L}}\left(m_{\phi}, m_{\phi^{\prime}}, \mu\right)+\frac{5}{6} \mathcal{M}^{4}\left(m_{\phi}, m_{\phi^{\prime}}\right)\right]\right\} \\
& +\frac{15}{8 M_{B}} \frac{g_{\Delta N}^{2}}{(4 \pi f)^{2}}\left\{\sum_{\phi} B_{\phi}^{B}\left[\overline{\mathcal{L}}\left(m_{\phi}, \mu\right)+\frac{9}{10} m_{\phi}^{4}\right]+\sum_{\phi \phi^{\prime}} B_{\phi \phi^{\prime}}^{B}\left[\overline{\mathcal{L}}\left(m_{\phi}, m_{\phi^{\prime}}, \mu\right)+\frac{9}{10} \mathcal{M}^{4}\left(m_{\phi}, m_{\phi^{\prime}}\right)\right]\right\} \\
& +\frac{6 \sigma_{M}^{(P Q)} \operatorname{str}\left(m_{Q}\right)}{(4 \pi f)^{2}}\left\{\sum_{\phi} A_{\phi}^{B}\left[\mathcal{L}\left(m_{\phi}, \mu\right)+\frac{2}{3} m_{\phi}^{2}\right]+\sum_{\phi \phi^{\prime}} A_{\phi \phi^{\prime}}^{B}\left[\mathcal{L}\left(m_{\phi}, m_{\phi^{\prime}}, \mu\right)+\frac{2}{3} \mathcal{M}^{2}\left(m_{\phi}, m_{\phi^{\prime}}\right)\right]\right\} \\
& +\frac{6 g_{\Delta N}^{2} \bar{\sigma}_{M} \operatorname{str}\left(m_{Q}\right)}{(4 \pi f)^{2}}\left\{\sum_{\phi} B_{\phi}^{B}\left[\mathcal{J}\left(m_{\phi}, \Delta, \mu\right)+m_{\phi}^{2}\right]+\sum_{\phi \phi^{\prime}} B_{\phi \phi^{\prime}}^{B}\left[\mathcal{J}\left(m_{\phi}, m_{\phi^{\prime}}, \Delta, \mu\right)+\mathcal{M}^{2}\left(m_{\phi}, m_{\phi^{\prime}}\right)\right]\right\} \\
& +\frac{3}{(4 \pi f)^{2}}\left\{\sum_{\phi} F_{\phi}^{B}\left[\mathcal{L}\left(m_{\phi}, \mu\right)+\frac{2}{3} m_{\phi}^{2}\right]+\sum_{\phi \phi^{\prime}} F_{\phi \phi^{\prime}}^{B}\left[\mathcal{L}\left(m_{\phi}, m_{\phi^{\prime}}, \mu\right)+\frac{2}{3} \mathcal{M}^{2}\left(m_{\phi}, m_{\phi^{\prime}}\right)\right]\right\} \\
& +\frac{2 g_{\Delta N}^{2} \gamma_{M}}{(4 \pi f)^{2}}\left\{\sum_{\phi} G_{\phi}^{B}\left[\mathcal{J}\left(m_{\phi}, \Delta, \mu\right)+m_{\phi}^{2}\right]+\sum_{\phi \phi^{\prime}} G_{\phi \phi^{\prime}}^{B}\left[\mathcal{J}\left(m_{\phi}, m_{\phi^{\prime}}, \Delta, \mu\right)+\mathcal{M}^{2}\left(m_{\phi}, m_{\phi^{\prime}}\right)\right]\right\}
\end{aligned}
$$

The above expression is written quite compactly. It involves the tree-level coefficients $m_{B}^{\prime}, m_{B}^{\prime \prime},\left(m^{2}\right)_{B},\left(m^{2}\right)_{B}^{\prime}$, $\left(\mathrm{mm}^{\prime}\right)_{B}$, and $\left(\mathrm{mm}^{\prime}\right)_{B}^{\prime}$. These are listed in Table $\mathbb{V}$ for the nucleon states labeled by $B$. The wavefunction renormalization $Z_{B}$ appearing in the above expression is given by

$$
\begin{aligned}
Z_{B}-1= & -\frac{3}{(4 \pi f)^{2}}\left\{\sum_{\phi} A_{\phi}^{B}\left[\mathcal{L}\left(m_{\phi}, \mu\right)+\frac{2}{3} m_{\phi}^{2}\right]+\sum_{\phi \phi^{\prime}} A_{\phi \phi^{\prime}}^{B}\left[\mathcal{L}\left(m_{\phi}, m_{\phi^{\prime}}, \mu\right)+\frac{2}{3} \mathcal{M}^{2}\left(m_{\phi}, m_{\phi^{\prime}}\right)\right]\right\} \\
& -\frac{3 g_{\Delta N}^{2}}{(4 \pi f)^{2}}\left\{\sum_{\phi} B_{\phi}^{B}\left[\mathcal{J}\left(m_{\phi}, \Delta, \mu\right)+m_{\phi}^{2}\right]+\sum_{\phi \phi^{\prime}} B_{\phi \phi^{\prime}}^{B}\left[\mathcal{J}\left(m_{\phi}, m_{\phi^{\prime}}, \Delta, \mu\right)+\mathcal{M}^{2}\left(m_{\phi}, m_{\phi^{\prime}}\right)\right]\right\}
\end{aligned}
$$

Furthermore we have made use of abbreviations for the new non-analytic functions arising from loop integrals in Eqs. (A38) and (A39). These are defined to be

$$
\begin{aligned}
\mathcal{L}\left(m_{\phi}, m_{\phi^{\prime}}, \mu\right) & =\mathcal{H}_{\phi \phi^{\prime}}\left[\mathcal{L}\left(m_{\phi}, \mu\right), \mathcal{L}\left(m_{\phi^{\prime}}, \mu\right), \mathcal{L}\left(m_{X}, \mu\right)\right], \\
\overline{\mathcal{L}}\left(m_{\phi}, m_{\phi^{\prime}}, \mu\right) & =\mathcal{H}_{\phi \phi^{\prime}}\left[\overline{\mathcal{L}}\left(m_{\phi}, \mu\right), \overline{\mathcal{L}}\left(m_{\phi^{\prime}}, \mu\right), \overline{\mathcal{L}}\left(m_{X}, \mu\right)\right] \\
\mathcal{J}\left(m_{\phi}, m_{\phi^{\prime}}, \delta, \mu\right) & =\mathcal{H}_{\phi \phi^{\prime}}\left[\mathcal{J}\left(m_{\phi}, \delta, \mu\right), \mathcal{J}\left(m_{\phi^{\prime}}, \delta, \mu\right), \mathcal{J}\left(m_{X}, \delta, \mu\right)\right]
\end{aligned}
$$

and arise from hairpin contributions. The various coefficients in the above sums over loop mesons and loop pairs of flavor-neutral mesons are listed in Tables $\mathbf{D}] \mathbf{X}$ One may check that in the limit $m_{j} \rightarrow m_{u}, m_{l} \rightarrow m_{d}$ the $\chi \mathrm{PT}$ results are obtained from the PQ $\chi \mathrm{PT}$ expressions with the proper matching of coefficients, see Eq. (A27)- (A34). 


\section{Deltas}

The relevant diagrams needed to obtain the delta masses at NNLO in PQ $\chi \mathrm{PT}$ are depicted in [27]. These include hairpin contributions from the flavor-diagonal propagator. To leading order in $\mathrm{PQ} \chi \mathrm{PT}$ the delta masses are

$$
M_{T}^{(1)}=\frac{2}{3} \gamma_{M} m_{T}-2 \bar{\sigma}_{M} \operatorname{str}\left(m_{Q}\right),
$$

where the coefficients $m_{T}$ are listed for deltas $T$ in Table At next-to-leading order, we have

$$
\begin{aligned}
M_{T}^{(3 / 2)} & =-\frac{5 g_{\Delta \Delta}^{2}}{72 \pi f^{2}}\left[\sum_{\phi} A_{\phi}^{T} m_{\phi}^{3}+\sum_{\phi \phi^{\prime}} A_{\phi \phi^{\prime}}^{T} \mathcal{M}^{3}\left(m_{\phi}, m_{\phi^{\prime}}\right)\right] \\
& -\frac{g_{\Delta N}^{2}}{(4 \pi f)^{2}}\left[\sum_{\phi} B_{\phi}^{T} \mathcal{F}\left(m_{\phi},-\Delta, \mu\right)+\sum_{\phi \phi^{\prime}} B_{\phi \phi^{\prime}}^{T} \mathcal{F}\left(m_{\phi}, m_{\phi^{\prime}},-\Delta, \mu\right)\right],
\end{aligned}
$$

where $\mathcal{F}(m, \Delta, \mu)$ is given by Eq. (21) and the functions $\mathcal{M}^{n}\left(m_{\phi}, m_{\phi^{\prime}}\right)$ and $\mathcal{F}\left(m_{\phi}, m_{\phi^{\prime}}, \delta, \mu\right)$ appear in Eq. (A37). The coefficients $A_{\phi}^{T}, A_{\phi \phi^{\prime}}^{T}, B_{\phi}^{T}$ and $B_{\phi \phi^{\prime}}^{T}$ appear in Table XI

At next-to-next-to leading order, we have the following contribution to the delta masses

$$
\begin{aligned}
& M_{T}^{(2)}=\left(Z_{T}-1\right) M_{T}^{(1)} \\
& +\frac{1}{4 \pi f}\left[\frac{t_{1}^{M}}{3}\left(m^{2}\right)_{T}+\frac{t_{2}^{M}}{3}\left(m m^{\prime}\right)_{T}+t_{3}^{M} \operatorname{str}\left(m_{Q}^{2}\right)+\frac{t_{4}^{M}}{3} m_{T} \operatorname{str}\left(m_{Q}\right)+t_{5}^{M}\left[\operatorname{str}\left(m_{Q}\right)\right]^{2}\right] \\
& -\frac{2 \gamma_{M}}{(4 \pi f)^{2}}\left[\sum_{\phi} C_{\phi}^{T} \mathcal{L}\left(m_{\phi}, \mu\right)+\sum_{\phi \phi^{\prime}} C_{\phi \phi^{\prime}}^{T} \mathcal{L}\left(m_{\phi}, m_{\phi^{\prime}}, \mu\right)\right] \\
& +\frac{2 \bar{\sigma}_{M}}{(4 \pi f)^{2}}\left[\sum_{\phi} \bar{C}_{\phi} \mathcal{L}\left(m_{\phi}, \mu\right)+\sum_{\phi \phi^{\prime}} \bar{C}_{\phi \phi^{\prime}} \mathcal{L}\left(m_{\phi}, m_{\phi^{\prime}}, \mu\right)\right] \\
& +\frac{1}{(4 \pi f)^{3}} \sum_{\phi}\left(t_{1}^{A(P Q)} D_{\phi}^{T}+t_{2}^{A} E_{\phi}^{T}+t_{3}^{A(P Q)} \bar{D}_{\phi}\right) \overline{\mathcal{L}}\left(m_{\phi}, \mu\right) \\
& +\frac{1}{(4 \pi f)^{3}} \sum_{\phi \phi^{\prime}}\left(t_{1}^{A(P Q)} D_{\phi \phi^{\prime}}^{T}+t_{2}^{A} E_{\phi \phi^{\prime}}^{T}+t_{3}^{A(P Q)} \bar{D}_{\phi \phi^{\prime}}\right) \overline{\mathcal{L}}\left(m_{\phi}, m_{\phi^{\prime}}, \mu\right) \\
& +\frac{1}{4(4 \pi f)^{3}} \sum_{\phi}\left[\left(t_{1}^{\tilde{A}(P Q)}+t_{1}^{v A(P Q)}\right) D_{\phi}^{T}+\left(t_{2}^{\tilde{A}}+t_{2}^{v A}\right) E_{\phi}^{T}+\left(t_{3}^{\tilde{A}(P Q)}+t_{3}^{v A(P Q)}\right) \bar{D}_{\phi}\right] \\
& \times\left[\overline{\mathcal{L}}\left(m_{\phi}, \mu\right)-\frac{1}{2} m_{\phi}^{4}\right] \\
& +\frac{1}{4(4 \pi f)^{3}} \sum_{\phi \phi^{\prime}}\left[\left(t_{1}^{A(\tilde{P} Q)}+t_{1}^{v A(P Q)}\right) D_{\phi \phi^{\prime}}^{T}+\left(t_{2}^{\tilde{A}(P Q)}+t_{2}^{v A}\right) E_{\phi \phi^{\prime}}^{T}+\left(t_{3}^{\tilde{A}(P Q)}+t_{3}^{v A}\right) \bar{D}_{\phi \phi^{\prime}}\right] \\
& \times\left[\overline{\mathcal{L}}\left(m_{\phi}, m_{\phi^{\prime}}, \mu\right)-\frac{1}{2} \mathcal{M}^{4}\left(m_{\phi}, m_{\phi^{\prime}}\right)\right] \\
& -\frac{5}{8} \frac{g_{\Delta \Delta}^{2}}{(4 \pi f)^{2} M_{B}}\left\{\sum_{\phi} A_{\phi}^{T}\left[\overline{\mathcal{L}}\left(m_{\phi}, \mu\right)+\frac{19}{10} m_{\phi}^{4}\right]+\sum_{\phi \phi^{\prime}} A_{\phi \phi^{\prime}}^{T}\left[\overline{\mathcal{L}}\left(m_{\phi}, m_{\phi^{\prime}}, \mu\right)+\frac{19}{10} \mathcal{M}^{4}\left(m_{\phi}, m_{\phi^{\prime}}\right)\right]\right\} \\
& -\frac{15}{16} \frac{g_{\Delta N}^{2}}{(4 \pi f)^{2} M_{B}}\left\{\sum_{\phi} B_{\phi}^{T}\left[\overline{\mathcal{L}}\left(m_{\phi}, \mu\right)-\frac{1}{10} m_{\phi}^{4}\right]+\sum_{\phi \phi^{\prime}} B_{\phi \phi^{\prime}}^{T}\left[\overline{\mathcal{L}}\left(m_{\phi}, m_{\phi^{\prime}}, \mu\right)-\frac{1}{10} \mathcal{M}^{4}\left(m_{\phi}, m_{\phi^{\prime}}\right)\right]\right\} \\
& -\frac{10 g_{\Delta \Delta}^{2} \bar{\sigma}_{M} \operatorname{str}\left(m_{Q}\right)}{3(4 \pi f)^{2}}\left\{\sum_{\phi} A_{\phi}^{T}\left[\mathcal{L}\left(m_{\phi}, \mu\right)+\frac{26}{15} m_{\phi}^{2}\right]+\sum_{\phi \phi^{\prime}} A_{\phi \phi^{\prime}}^{T}\left[\mathcal{L}\left(m_{\phi}, m_{\phi^{\prime}}, \mu\right)+\frac{26}{15} \mathcal{M}^{2}\left(m_{\phi}, m_{\phi^{\prime}}\right)\right]\right\} \\
& -\frac{3 g_{\Delta N}^{2} \sigma_{M}^{(P Q)} \operatorname{str}\left(m_{Q}\right)}{(4 \pi f)^{2}}\left[\sum_{\phi} B_{\phi}^{T} \mathcal{J}\left(m_{\phi},-\Delta, \mu\right)+\sum_{\phi \phi^{\prime}} B_{\phi \phi^{\prime}}^{T} \mathcal{J}\left(m_{\phi}, m_{\phi^{\prime}},-\Delta, \mu\right)\right] \\
& +\frac{10 g_{\Delta \Delta}^{2} \gamma_{M}}{9(4 \pi f)^{2}}\left\{\sum_{\phi} F_{\phi}^{T}\left[\mathcal{L}\left(m_{\phi}, \mu\right)+\frac{26}{15} m_{\phi}^{2}\right]+\sum_{\phi \phi^{\prime}} F_{\phi \phi^{\prime}}^{T}\left[\mathcal{L}\left(m_{\phi}, m_{\phi^{\prime}}, \mu\right)+\frac{26}{15} \mathcal{M}^{2}\left(m_{\phi}, m_{\phi^{\prime}}\right)\right]\right\}
\end{aligned}
$$




$$
-\frac{3 g_{\Delta N}^{2}}{2(4 \pi f)^{2}}\left[\sum_{\phi} G_{\phi}^{T} \mathcal{J}\left(m_{\phi},-\Delta, \mu\right)+\sum_{\phi \phi^{\prime}} G_{\phi \phi^{\prime}}^{T} \mathcal{J}\left(m_{\phi}, m_{\phi^{\prime}},-\Delta, \mu\right)\right] .
$$

As with the NNLO contributions to the nucleon masses, the above expression is written quite compactly. It involves the tree-level coefficients $m_{T},\left(m^{2}\right)_{T}$, and $\left(\mathrm{mm}^{\prime}\right)_{T}$ which are listed in Table 1 for the delta states $T$. The NNLO result involves the wavefunction renormalization $Z_{T}$, which we find to be

$$
\begin{aligned}
Z_{T}-1= & -\frac{5 g_{\Delta \Delta}^{2}}{3(4 \pi f)^{2}}\left\{\sum_{\phi} A_{\phi}^{T}\left[\mathcal{L}\left(m_{\phi}, \mu\right)+\frac{26}{15} m_{\phi}^{2}\right]+\sum_{\phi \phi^{\prime}} A_{\phi \phi^{\prime}}^{T}\left[\mathcal{L}\left(m_{\phi}, m_{\phi^{\prime}}, \mu\right)+\frac{26}{15} \mathcal{M}^{2}\left(m_{\phi}, m_{\phi^{\prime}}\right)\right]\right\} \\
& -\frac{3 g_{\Delta N}^{2}}{2(4 \pi f)^{2}}\left[\sum_{\phi} B_{\phi}^{T} \mathcal{J}\left(m_{\phi},-\Delta, \mu\right)+\sum_{\phi \phi^{\prime}} B_{\phi \phi^{\prime}}^{T} \mathcal{J}\left(m_{\phi}, m_{\phi^{\prime}},-\Delta, \mu\right)\right] .
\end{aligned}
$$

The functions arising from hairpin contributions are listed in Eq. A40. The various coefficients in the above sums are listed in Tables XI XV] Finally, one can check that the $\chi$ PT result is recovered in the limit $m_{j} \rightarrow m_{u}, m_{l} \rightarrow m_{d}$, and by utilizing the matching relations given in Eqs. (A27), A31), A33) and (A34).

[1] J. Gasser and H. Leutwyler, Ann. Phys. 158, 142 (1984).

[2] C. W. Bernard et al., Phys. Rev. D64, 054506 (2001), hep-lat/0104002.

[3] A. Ali Khan et al. (CP-PACS), Phys. Rev. D65, 054505 (2002), hep-lat/0105015.

[4] S. Aoki et al. (JLQCD), Phys. Rev. D68, 054502 (2003), hep-lat/0212039.

[5] J. M. Zanotti et al. (CSSM Lattice), Phys. Rev. D68, 054506 (2003), hep-lat/0304001.

[6] V. Bernard, T. R. Hemmert, and U.-G. Meissner, Nucl. Phys. A732, 149 (2004), hep-ph/0307115.

[7] C. Aubin et al., Phys. Rev. D70, 094505 (2004), hep-lat/0402030.

[8] D. Toussaint and C. T. H. Davies, Nucl. Phys. Proc. Suppl. 140, 234 (2005), hep-lat/0409129.

[9] D. B. Renner et al. (LHP), Nucl. Phys. Proc. Suppl. 140, 255 (2005), hep-lat/0409130.

[10] U. M. Heller (2004), hep-ph/0412295.

[11] D. B. Leinweber, A. W. Thomas, and R. D. Young, Phys. Rev. Lett. 92, 242002 (2004), hep-lat/0302020.

[12] M. Procura, T. R. Hemmert, and W. Weise, Phys. Rev. D69, 034505 (2004), hep-lat/0309020.

[13] A. Ali Khan et al. (QCDSF-UKQCD), Nucl. Phys. B689, 175 (2004), hep-lat/0312030.

[14] U.-G. Meissner (2005), hep-ph/0501009.

[15] C. Bernard et al., Nucl. Phys. Proc. Suppl. 119, 170 (2003), hep-lat/0209086.

[16] S. R. Beane, Nucl. Phys. B695, 192 (2004), hep-lat/0403030.

[17] J. Gasser, Ann. Phys. 136, 62 (1981).

[18] E. Jenkins, Nucl. Phys. B368, 190 (1992).

[19] V. Bernard, N. Kaiser, and U. G. Meissner, Z. Phys. C60, 111 (1993), hep-ph/9303311.

[20] R. F. Lebed, Nucl. Phys. B430, 295 (1994), hep-ph/9311234.

[21] R. F. Lebed and M. A. Luty, Phys. Lett. B329, 479 (1994), hep-ph/9401232.

[22] M. K. Banerjee and J. Milana, Phys. Rev. D52, 6451 (1995), hep-ph/9410398.

[23] B. Borasoy and U.-G. Meissner, Annals Phys. 254, 192 (1997), hep-ph/9607432.

[24] T. Becher and H. Leutwyler, Eur. Phys. J. C9, 643 (1999), hep-ph/9901384.

[25] M. Frink and U.-G. Meissner, JHEP 07, 028 (2004), hep-lat/0404018.

[26] A. Walker-Loud, Nucl. Phys. A747, 476 (2005), hep-lat/0405007.

[27] B. C. Tiburzi and A. Walker-Loud, Nucl. Phys. A748, 513 (2005), hep-lat/0407030.

[28] B. C. Lehnhart, J. Gegelia, and S. Scherer, J. Phys. G31, 89 (2005), hep-ph/0412092.

[29] E. Jenkins and A. V. Manohar (1991), talk presented at the Workshop on Effective Field Theories of the Standard Model, Dobogoko, Hungary, Aug 1991.

[30] E. Jenkins and A. V. Manohar, Phys. Lett. B255, 558 (1991).

[31] E. Jenkins and A. V. Manohar, Phys. Lett. B259, 353 (1991).

[32] V. Bernard, N. Kaiser, J. Kambor, and U. G. Meissner, Nucl. Phys. B388, 315 (1992).

[33] V. Bernard, N. Kaiser, and U.-G. Meissner, Int. J. Mod. Phys. E4, 193 (1995), hep-ph/9501384.

[34] T. R. Hemmert, B. R. Holstein, and J. Kambor, Phys. Lett. B395, 89 (1997), hep-ph/9606456.

[35] T. R. Hemmert, B. R. Holstein, and J. Kambor, J. Phys. G24, 1831 (1998), hep-ph/9712496.

[36] M. E. Luke and A. V. Manohar, Phys. Lett. B286, 348 (1992), hep-ph/9205228.

[37] A. V. Manohar and M. B. Wise, Cambridge Monogr. Part. Phys. Nucl. Phys. Cosmol. 10, 1 (2000).

[38] J. Gasser and H. Leutwyler, Nucl. Phys. B94, 269 (1975).

[39] A. Duncan, E. Eichten, and H. Thacker, Phys. Rev. Lett. 76, 3894 (1996), hep-lat/9602005.

[40] A. Duncan, E. Eichten, and H. Thacker, Phys. Lett. B409, 387 (1997), hep-lat/9607032.

[41] A. Duncan, E. Eichten, and R. Sedgewick (2004), hep-lat/0405014. 
TABLE V: The tree-level coefficients in $\chi \mathrm{PT}$ and PQ $\chi \mathrm{PT}$. The coefficients $m_{B}, m_{B}^{\prime}, m_{B}^{\prime \prime},\left(m^{2}\right)_{B},\left(m^{2}\right)_{B}^{\prime},\left(m m^{\prime}\right)_{B}$, and $\left(m m^{\prime}\right)_{B}^{\prime}$ are listed for the nucleons $B$.

\begin{tabular}{c|ccccccc} 
& $m_{B}$ & $m_{B}^{\prime}$ & $m_{B}^{\prime \prime}$ & $\left(m^{2}\right)_{B}$ & $\left(m^{2}\right)_{B}^{\prime}$ & $\left(m m^{\prime}\right)_{B}$ & $\left(m m^{\prime}\right)_{B}^{\prime}$ \\
\hline$p$ & $m_{u}$ & $m_{u}+2 m_{d}$ & $5 m_{u}+m_{d}$ & $m_{u}^{2}+2 m_{d}^{2}$ & $5 m_{u}^{2}+m_{d}^{2}$ & $m_{u}^{2}+5 m_{u} m_{d}$ & $m_{u}^{2}-4 m_{u} m_{d}$ \\
$n$ & $m_{d}$ & $2 m_{u}+m_{d}$ & $m_{u}+5 m_{d}$ & $2 m_{u}^{2}+m_{d}^{2}$ & $m_{u}^{2}+5 m_{d}^{2}$ & $5 m_{u} m_{d}+m_{d}^{2}$ & $-4 m_{u} m_{d}+m_{d}^{2}$
\end{tabular}

[42] J. Gasser, A. Rusetsky, and I. Scimemi, Eur. Phys. J. C32, 97 (2003), hep-ph/0305260.

[43] G. A. Miller, B. M. K. Nefkens, and I. Slaus, Phys. Rept. 194, 1 (1990).

[44] C. W. Bernard and M. F. L. Golterman, Phys. Rev. D49, 486 (1994), hep-lat/9306005.

[45] S. R. Sharpe, Phys. Rev. D56, 7052 (1997), hep-lat/9707018.

[46] M. F. L. Golterman and K.-C. Leung, Phys. Rev. D57, 5703 (1998), hep-lat/9711033.

[47] S. R. Sharpe and N. Shoresh, Phys. Rev. D62, 094503 (2000), hep-lat/0006017.

[48] S. R. Sharpe and N. Shoresh, Phys. Rev. D64, 114510 (2001), hep-lat/0108003.

[49] S. R. Sharpe and R. S. Van de Water, Phys. Rev. D69, 054027 (2004), hep-lat/0310012.

[50] J.-W. Chen and M. J. Savage, Phys. Rev. D65, 094001 (2002), hep-lat/0111050.

[51] S. R. Beane and M. J. Savage, Nucl. Phys. A709, 319 (2002), hep-lat/0203003.

[52] B. C. Tiburzi, Phys. Rev. D71, 034501 (2005), hep-lat/0410033.

[53] J. N. Labrenz and S. R. Sharpe, Phys. Rev. D54, 4595 (1996), hep-lat/9605034.

\section{APPENDIX B: PARTIALLY QUENCHED COEFFICIENT TABLES}

Here we list the tables needed for the partially quenched calculation which have not already been listed in the text. One can obtain from the authors a Mathematica notebook which evaluates PQ $\chi \mathrm{PT}$ and $\chi \mathrm{PT}$ expressions for the nucleon and delta masses in various limits. We consider the case of non-degenerate valence and sea quarks, as well as non-degenerate valence and degenerate sea quarks and further degenerate valence and sea quarks. 
TABLE VI: The coefficients $A_{\phi}^{B}, A_{\phi \phi^{\prime}}^{B}, B_{\phi}^{B}$, and $B_{\phi \phi^{\prime}}^{T}$ in PQ $\chi$ PT. Coefficients are listed for the nucleons $B$, and for $A_{\phi}^{B}$ and $B_{\phi}^{B}$ are grouped into contributions from loop mesons with mass $m_{\phi}$, while for $A_{\phi \phi^{\prime}}^{T}$ and $B_{\phi \phi^{\prime}}^{B}$ are grouped into contributions from pairs of quark-basis $\eta_{q}$ mesons.

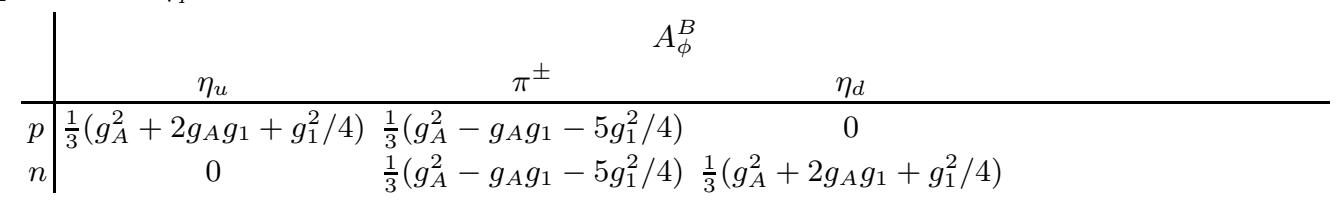

\begin{tabular}{c|cccc} 
& $j u$ & $l u$ & $j d$ & $l d$ \\
\hline$p$ & $\frac{1}{3}\left(2 g_{A}^{2}+g_{A} g_{1}+g_{1}^{2} / 2\right)$ & $\frac{1}{3}\left(2 g_{A}^{2}+g_{A} g_{1}+g_{1}^{2} / 2\right)$ & $g_{1}^{2} / 4$ & $g_{1}^{2} / 4$ \\
$n$ & $g_{1}^{2} / 4$ & $g_{1}^{2} / 4$ & $\frac{1}{3}\left(2 g_{A}^{2}+g_{A} g_{1}+g_{1}^{2} / 2\right)$ & $\frac{1}{3}\left(2 g_{A}^{2}+g_{A} g_{1}+g_{1}^{2} / 2\right)$
\end{tabular}

\begin{tabular}{c|ccc} 
& \multicolumn{3}{|c}{$A_{\phi \phi^{\prime}}^{B}$} \\
\hline$p$ & $\eta_{u} \eta_{u}$ & $\eta_{u} \eta_{d}$ & $\eta_{d} \eta_{d}$ \\
\hline$n$ & $g_{A}^{2}+g_{A} g_{1}+g_{1}^{2} / 4$ & $g_{A} g_{1}+g_{1}^{2} / 2$ & $g_{1}^{2} / 4$ \\
$g_{1}^{2} / 4$ & $g_{A} g_{1}+g_{1}^{2} / 2$ & $g_{A}^{2}+g_{A} g_{1}+g_{1}^{2} / 4$
\end{tabular}

\begin{tabular}{c|cccc} 
& \multicolumn{3}{|c}{$B_{\phi}^{B}$} \\
\hline$p$ & $\eta_{u}$ & $\pi^{ \pm}$ & & $\eta_{d}$ \\
\hline$n$ & $\frac{1}{9}$ & $\frac{5}{9}$ & 0 \\
0 & 0 & $\frac{5}{9}$ & $\frac{1}{9}$
\end{tabular}

\begin{tabular}{c|cccc} 
& $j u$ & $l u$ & $j d$ & $l d$ \\
\hline$p$ & $\frac{1}{9}$ & $\frac{1}{9}$ & $\frac{2}{9}$ & $\frac{2}{9}$ \\
$n$ & $\frac{2}{9}$ & $\frac{2}{9}$ & $\frac{1}{9}$ & $\frac{1}{9}$
\end{tabular}

\begin{tabular}{c|ccc} 
& \multicolumn{3}{|c}{$B_{\phi \phi^{\prime}}^{B}$} \\
\hline$p$ & $\eta_{u} \eta_{u}$ & $\eta_{u} \eta_{d}$ & $\eta_{d} \eta_{d}$ \\
$n$ & $\frac{2}{9}$ & $-\frac{4}{9}$ & $\frac{2}{9}$ \\
$\frac{2}{9}$ & $-\frac{4}{9}$ & $\frac{2}{9}$
\end{tabular}


TABLE VII: The coefficients $C_{\phi}^{B}, C_{\phi \phi^{\prime}}^{B}, \bar{C}_{\phi}, \bar{C}_{\phi \phi^{\prime}}, D_{\alpha, \phi}^{B}, D_{\alpha, \phi \phi^{\prime}}^{B}, D_{\beta, \phi}^{B}, D_{\beta, \phi \phi^{\prime}}^{B}, \bar{D}_{\phi}$, and $\bar{D}_{\phi \phi^{\prime}}$ in PQ $\chi$ PT. The coefficients $\bar{C}_{\phi}$, $\bar{C}_{\phi \phi^{\prime}}, \bar{D}_{\phi}$ and $\bar{D}_{\phi \phi^{\prime}}$ are identical for all nucleons and deltas. The remaining coefficients are listed for nucleon states $B$, and for $C_{\phi}^{B}$, and $D_{\phi}^{B}$ are grouped into contributions from loop mesons with mass $m_{\phi}$, while for $C_{\phi \phi^{\prime}}^{B}$ and $D_{\phi \phi^{\prime}}^{B}$ are grouped into contributions from pairs of quark-basis $\eta_{q}$ mesons. If a particular meson or pair of flavor-neutral mesons is not listed, then the value of the coefficient is zero for the nucleons.

$$
\begin{aligned}
& \begin{array}{c|cccc} 
& \multicolumn{4}{|c}{C_{\phi}^{B}} \\
& j u & l u & j d & l d \\
\hline p & \frac{1}{3}\left(5 \alpha_{M}^{(P Q)}+2 \beta_{M}^{(P Q)}\right) & \frac{1}{3}\left(5 \alpha_{M}^{(P Q)}+2 \beta_{M}^{(P Q)}\right) & \frac{1}{3}\left(\alpha_{M}^{(P Q)}+4 \beta_{M}^{(P Q)}\right) & \frac{1}{3}\left(\alpha_{M}^{(P Q)}+4 \beta_{M}^{(P Q)}\right) \\
& \times\left(m_{u}+m_{j}\right) & \times\left(m_{u}+m_{l}\right) & \times\left(m_{d}+m_{j}\right) & \times\left(m_{d}+m_{l}\right) \\
& & & & \\
& & & \\
& & & \\
& \frac{1}{3}\left(\alpha_{M}^{(P Q)}+4 \beta_{M}^{(P Q)}\right) & \frac{1}{3}\left(\alpha_{M}^{(P Q)}+4 \beta_{M}^{(P Q)}\right) & \frac{1}{3}\left(5 \alpha_{M}^{(P Q)}+2 \beta_{M}^{(P Q)}\right) & \frac{1}{3}\left(5 \alpha_{M}^{(P Q)}+2 \beta_{M}^{(P Q)}\right) \\
& \times\left(m_{u}+m_{j}\right) & \times\left(m_{u}+m_{l}\right) & \times\left(m_{d}+m_{j}\right) & \times\left(m_{d}+m_{l}\right)
\end{array}
\end{aligned}
$$

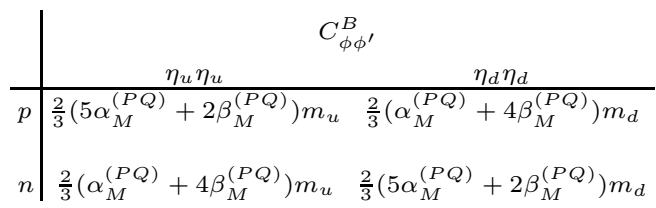

$$
\begin{aligned}
& \begin{array}{ccc|cc}
\multicolumn{3}{c|}{\bar{C}_{\phi}} & \multicolumn{2}{c}{\bar{C}_{\phi \phi^{\prime}}} \\
j j & j l & l l & \eta_{j} \eta_{j} & \eta_{l} \eta_{l} \\
\hline 2 m_{j} & 2\left(m_{j}+m_{l}\right) & 2 m_{l} & 2 m_{j} & 2 m_{l}
\end{array} \\
& \begin{array}{c|cccc|cc} 
& \multicolumn{4}{|c|}{D_{\alpha, \phi}^{B}} & \multicolumn{2}{c}{D_{\alpha, \phi \phi^{\prime}}^{B}} \\
& j u & l u & j d & l d & \eta_{u} \eta_{u} & \eta_{d} \eta_{d} \\
\hline p & \frac{5}{6} & \frac{5}{6} & \frac{1}{6} & \frac{1}{6} & \frac{5}{6} & \frac{1}{6} \\
n & \frac{1}{6} & \frac{1}{6} & \frac{5}{6} & \frac{5}{6} & \frac{1}{6} & \frac{5}{6}
\end{array} \\
& \begin{array}{c|cccc|cc} 
& \multicolumn{4}{|c|}{D_{\beta, \phi}^{B}} & \multicolumn{2}{c}{D_{\beta, \phi \phi^{\prime}}^{B}} \\
& j u & l u & j d & l d & \eta_{u} \eta_{u} & \eta_{d} \eta_{d} \\
\hline p & \frac{1}{3} & \frac{1}{3} & \frac{2}{3} & \frac{2}{3} & \frac{1}{3} & \frac{2}{3} \\
n & \frac{2}{3} & \frac{2}{3} & \frac{1}{3} & \frac{1}{3} & \frac{2}{3} & \frac{1}{3}
\end{array} \\
& \begin{array}{ccc|cc}
\multicolumn{3}{c|}{\bar{D}_{\phi}} & \multicolumn{2}{|c}{\bar{D}_{\phi \phi^{\prime}}} \\
j j & j l & l l & \eta_{j} \eta_{j} & \eta_{l} \eta_{l} \\
\hline 1 & 2 & 1 & 1 & 1
\end{array}
\end{aligned}
$$

\begin{tabular}{|c|c|c|c|c|c|c|}
\hline & & $E_{\phi}^{B}$ & & & $E_{\phi \phi^{\prime}}^{B}$ & \\
\hline & $\eta_{u}$ & $\pi^{ \pm}$ & $\eta_{d}$ & $\eta_{u} \eta_{u}$ & $\eta_{u} \eta_{d}$ & $\eta_{d} \eta_{d}$ \\
\hline$p$ & $\frac{1}{6}$ & $-\frac{2}{3}$ & 0 & $\frac{1}{6}$ & $\frac{5}{6}$ & 0 \\
\hline$n$ & 0 & $-\frac{2}{3}$ & $\frac{1}{6}$ & 0 & $\frac{5}{6}$ & $\frac{1}{6}$ \\
\hline & & $E_{\phi}^{\prime B}$ & & & $E_{\phi \phi^{\prime}}^{\prime B}$ & \\
\hline & $\eta_{u}$ & $\pi^{ \pm}$ & $\eta_{d}$ & $\eta_{u} \eta_{u}$ & $\eta_{u} \eta_{d}$ & $\eta_{d} \eta_{d}$ \\
\hline$p$ & $\frac{1}{6}$ & $\frac{5}{6}$ & 0 & $\frac{1}{6}$ & $-\frac{2}{3}$ & 0 \\
\hline$n$ & 0 & $\frac{5}{6}$ & $\frac{1}{6}$ & 0 & $-\frac{2}{3}$ & $\frac{1}{6}$ \\
\hline
\end{tabular}

TABLE VIII: The coefficients $E_{\phi}^{B}, E_{\phi \phi^{\prime}}^{B}, E_{\phi}^{\prime B}$ and $E_{\phi \phi^{\prime}}^{\prime B}$ in PQ $\chi \mathrm{PT}$. Coefficients are listed for the nucleons, and for $E_{\phi}^{B}$ and $E_{\phi}^{\prime B}$ are grouped into contributions from loop mesons with mass $m_{\phi}$, while for $E_{\phi \phi^{\prime}}^{B}$ and $E_{\phi \phi^{\prime}}^{\prime B}$ are grouped into contributions from pairs of quark-basis $\eta_{q}$ mesons. 
TABLE IX: The coefficients $F_{\phi}^{B}$ and $F_{\phi \phi^{\prime}}^{B}$ in PQ $\chi \mathrm{PT}$. Coefficients are listed for the nucleons, and for $F_{\phi}^{B}$ are grouped into contributions from loop mesons with mass $m_{\phi}$, while for $F_{\phi \phi^{\prime}}^{B}$ are grouped into contributions from pairs of quark-basis $\eta_{q}$ mesons.

\begin{tabular}{|c|c|c|c|c|}
\hline & $\eta_{u}$ & $F_{\phi}^{B}$ & $\eta_{d}$ & \\
\hline$p$ & $\begin{array}{l}\frac{1}{24}\left\{m_{u}\left[16 g_{A}^{2} \alpha_{M}^{(P Q)}\right.\right. \\
\quad+4 g_{A} g_{1}\left(7 \alpha_{M}^{(P Q)}+2 \beta_{M}^{(P Q)}\right) \\
\left.\quad+g_{1}^{2}\left(5 \alpha_{M}^{(P Q)}-2 \beta_{M}^{(P Q)}\right)\right] \\
+m_{d}\left[16 g_{A}^{2} \beta_{M}^{(P Q)}\right. \\
\quad+4 g_{A} g_{1}\left(\alpha_{M}^{(P Q)}+6 \beta_{M}^{(P Q)}\right) \\
\left.\left.\quad+g_{1}^{2}\left(6 \beta_{M}^{(P Q)}-\alpha_{M}^{(P Q)}\right)\right]\right\} \\
\end{array}$ & $\begin{array}{c}\frac{1}{24}\left\{m_{u}\left[16 g_{A}^{2} \beta_{M}^{(P Q)}\right.\right. \\
-4 g_{A} g_{1}\left(\alpha_{M}^{(P Q)}+2 \beta_{M}^{(P Q)}\right) \\
\left.-g_{1}^{2}\left(15 \alpha_{M}^{(P Q)}+14 \beta_{M}^{(P Q)}\right)\right] \\
+m_{d}\left[16 g_{A}^{2} \alpha_{M}^{(P Q)}\right. \\
-4 g_{A} g_{1}\left(3 \alpha_{M}^{(P Q)}+2 \beta_{M}^{(P Q)}\right) \\
\left.\left.-g_{1}^{2}\left(5 \alpha_{M}^{(P Q)}+6 \beta_{M}^{(P Q)}\right)\right]\right\} \\
\end{array}$ & 0 & \\
\hline$n$ & 0 & $\begin{array}{c}\frac{1}{24}\left\{m_{u}\left[16 g_{A}^{2} \alpha_{M}^{(P Q)}\right.\right. \\
-4 g_{A} g_{1}\left(3 \alpha_{M}^{(P Q)}+2 \beta_{M}^{(P Q)}\right) \\
\left.-g_{1}^{2}\left(5 \alpha_{M}^{(P Q)}+6 \beta_{M}^{(P Q)}\right)\right] \\
+m_{d}\left[16 g_{A}^{2} \beta_{M}^{(P Q)}\right. \\
\quad-4 g_{A} g_{1}\left(\alpha_{M}^{(P Q)}+2 \beta_{M}^{(P Q)}\right) \\
\left.\left.\quad-g_{1}^{2}\left(15 \alpha_{M}^{(P Q)}+14 \beta_{M}^{(P Q)}\right)\right]\right\} \\
\end{array}$ & $\begin{array}{l}\frac{1}{24}\left\{m_{u}\left[16 g_{A}^{2} \beta_{M}^{(P Q)}\right.\right. \\
\quad+4 g_{A} g_{1}\left(\alpha_{M}^{(P Q)}+6 \beta_{M}^{(P Q)}\right) \\
\left.\quad+g_{1}^{2}\left(6 \beta_{M}^{(P Q)}-\alpha_{M}^{(P Q)}\right)\right] \\
+m_{d}\left[16 g_{A}^{2} \alpha_{M}^{(P Q)}\right. \\
\quad+4 g_{A} g_{1}\left(7 \alpha_{M}^{(P Q)}+2 \beta_{M}^{(P Q)}\right) \\
\left.\left.\quad+g_{1}^{2}\left(5 \alpha_{M}^{(P Q)}-2 \beta_{M}^{(P Q)}\right)\right]\right\} \\
\end{array}$ & \\
\hline & $j u$ & $l u$ & $j d$ & $l d$ \\
\hline$\overline{p p}$ & $\begin{array}{c}\frac{1}{24}\left\{m_{u}\left[8 g_{A}^{2}\left(\alpha_{M}^{(P Q)}+2 \beta_{M}^{(P Q)}\right)\right.\right. \\
+4 g_{A} g_{1}\left(\alpha_{M}^{(P Q)}+2 \beta_{M}^{(P Q)}\right) \\
\left.\quad+g_{1}^{2}\left(3 \alpha_{M}^{(P Q)}+2 \beta_{M}^{(P Q)}\right)\right] \\
+2 m_{j}\left[8 g_{A}^{2} \alpha_{M}^{(P Q)}\right. \\
\quad+4 g_{A} g_{1} \alpha_{M}^{(P Q)} \\
\left.\quad+g_{1}^{2}\left(\alpha_{M}^{(P Q)}+2 \beta_{M}^{(P Q)}\right)\right] \\
+m_{d}\left[8 g_{A}^{2}\left(\alpha_{M}^{(P Q)}+2 \beta_{M}^{(P Q)}\right)\right. \\
\quad+4 g_{A} g_{1}\left(\alpha_{M}^{(P Q)}+2 \beta_{M}^{(P Q)}\right) \\
\left.\left.\quad+g_{1}^{2}\left(3 \alpha_{M}^{(P Q)}+2 \beta_{M}^{(P Q)}\right)\right]\right\} \\
\end{array}$ & $\begin{array}{c}\frac{1}{24}\left\{m_{u}\left[8 g_{A}^{2}\left(\alpha_{M}^{(P Q)}+2 \beta_{M}^{(P Q)}\right)\right.\right. \\
+4 g_{A} g_{1}\left(\alpha_{M}^{(P Q)}+2 \beta_{M}^{(P Q)}\right) \\
\left.+g_{1}^{2}\left(3 \alpha_{M}^{(P)}+2 \beta_{M}^{(P Q)}\right)\right] \\
+m_{d}\left[8 g_{A}^{2}\left(\alpha_{M}^{(P Q)}+2 \beta_{M}^{(P Q)}\right)\right. \\
+4 g_{A} g_{1}\left(\alpha_{M}^{(P Q)}+2 \beta_{M}^{(P Q)}\right) \\
\left.\quad+g_{1}^{2}\left(3 \alpha_{M}^{(P Q)}+2 \beta_{M}^{(P Q)}\right)\right] \\
+2 m_{l}\left[8 g_{A}^{2} \alpha_{M}^{(P Q)}\right. \\
\left.\left.+4 g_{A} g_{1} \alpha_{M}^{(P Q)}\right]\right\} \\
\left.\left.+g_{1}^{2}\left(\alpha_{M}^{(P Q)}+2 \beta_{M}^{(P Q)}\right)\right]\right\} \\
\end{array}$ & $\begin{array}{c}\frac{1}{12} g_{1}^{2}\left[m_{u}\left(5 \alpha_{M}^{(P Q)}+2 \beta_{M}^{(P Q)}\right)\right. \\
\left.\quad+m_{j}\left(\alpha_{M}^{(P Q)}+4 \beta_{M}^{(P)}\right)\right]\end{array}$ & $\begin{array}{c}\frac{1}{12} g_{1}^{2}\left[m_{u}\left(5 \alpha_{M}^{(P Q)}+2 \beta_{M}^{(P Q)}\right)\right. \\
\left.+m_{l}\left(\alpha_{M}^{(P Q)}+4 \beta_{M}^{(P Q)}\right)\right] \\
\\
\end{array}$ \\
\hline$n$ & $\begin{array}{l}\frac{1}{12} g_{1}^{2}\left[m_{d}\left(5 \alpha_{M}^{(P Q)}+2 \beta_{M}^{(P Q)}\right)\right. \\
\left.\quad+m_{j}\left(\alpha_{M}^{(P Q)}+4 \beta_{M}^{(P Q)}\right)\right]\end{array}$ & $\begin{array}{l}\frac{1}{12} g_{1}^{2}\left[m_{d}\left(5 \alpha_{M}^{(P Q)}+2 \beta_{M}^{(P Q)}\right)\right. \\
\left.\quad+m_{l}\left(\alpha_{M}^{(P Q)}+4 \beta_{M}^{(P Q)}\right)\right]\end{array}$ & $\begin{array}{c}\frac{1}{24}\left\{m_{u}\left[8 g_{A}^{2}\left(\alpha_{M}^{(P Q)}+2 \beta_{M}^{(P Q)}\right)\right.\right. \\
+4 g_{A} g_{1}\left(\alpha_{M}^{(P Q)}+2 \beta_{M}^{(P Q)}\right) \\
\left.+g_{1}^{2}\left(3 \alpha_{M}^{(P Q)}+2 \beta_{M}^{(P Q)}\right)\right] \\
+2 m_{j}\left[8 g_{A}^{2} \alpha_{M}^{(P Q)}\right. \\
\quad+4 g_{A} g_{1} \alpha_{M}^{(P Q)} \\
\left.\quad+g_{1}^{2}\left(\alpha_{M}^{(P Q)}+2 \beta_{M}^{(P Q)}\right)\right] \\
+m_{d}\left[8 g_{A}^{2}\left(\alpha_{M}^{(P Q)}+2 \beta_{M}^{(P Q)}\right)\right. \\
+4 g_{A} g_{1}\left(\alpha_{M}^{(P Q)}+2 \beta_{M}^{(P Q)}\right) \\
\left.\left.\quad+g_{1}^{2}\left(3 \alpha_{M}^{(P Q)}+2 \beta_{M}^{(P Q)}\right)\right]\right\} \\
\end{array}$ & $\begin{array}{c}\frac{1}{24}\left\{m_{u}\left[8 g_{A}^{2}\left(\alpha_{M}^{(P Q)}+2 \beta_{M}^{(P Q)}\right)\right.\right. \\
\quad+4 g_{A} g_{1}\left(\alpha_{M}^{(P Q)}+2 \beta_{M}^{(P Q)}\right) \\
\left.\quad+g_{1}^{2}\left(3 \alpha_{M Q)}^{(P Q)}+2 \beta_{M}^{(P Q)}\right)\right] \\
+m_{d}\left[8 g_{A}^{2}\left(\alpha_{M}^{(P Q)}+2 \beta_{M}^{(P Q)}\right)\right. \\
\quad+4 g_{A} g_{1}\left(\alpha_{M}^{(P Q)}+2 \beta_{M}^{(P Q)}\right) \\
\left.\quad+g_{1}^{2}\left(3 \alpha_{M}^{(P Q)}+2 \beta_{M}^{(P Q)}\right)\right] \\
+2 m_{l}\left[8 g_{A}^{2} \alpha_{M}^{(P Q)}\right. \\
\quad+4 g_{A} g_{1} \alpha_{M}^{(P Q)} \\
\left.\quad+g_{1}^{2}\left(\alpha_{M}^{(P Q)}+2 \beta_{M}^{(P Q)}\right)\right] \\
\end{array}$ \\
\hline \multicolumn{5}{|c|}{$F_{\phi \phi^{\prime}}^{B}$} \\
\hline & $\eta_{u} \eta_{u}$ & $\begin{array}{c}\eta_{u} \eta_{d} \\
\end{array}$ & $\eta_{d} \eta_{d}$ & \\
\hline p & $\begin{array}{l}\frac{1}{12}\left(2 g_{A}+g_{1}\right)^{2} \times \\
{\left[m_{u}\left(5 \alpha_{M}^{(P Q)}+2 \beta_{M}^{(P Q)}\right)\right.} \\
\left.\quad+m_{d}\left(\alpha_{M}^{(P Q)}+4 \beta_{M}^{(P Q)}\right)\right] \\
\end{array}$ & $\begin{array}{l}\frac{1}{6}\left(2 g_{A} g_{1}+g_{1}^{2}\right) \times \\
{\left[m_{u}\left(5 \alpha_{M}^{(P Q)}+2 \beta_{M}^{(P Q)}\right)\right.} \\
\left.\quad+m_{d}\left(\alpha_{M}^{(P Q)}+4 \beta_{M}^{(P Q)}\right)\right]\end{array}$ & $\begin{array}{r}\frac{1}{12} g_{1}^{2}\left[m_{u}\left(5 \alpha_{M}^{(P Q)}+2 \beta_{M}^{(P Q)}\right)\right. \\
\left.+m_{d}\left(\alpha_{M}^{(P Q)}+4 \beta_{M}^{(P Q)}\right)\right]\end{array}$ & \\
\hline$n$ & $\begin{array}{c}\frac{1}{12} g_{1}^{2}\left[m_{u}\left(\alpha_{M}^{(P Q)}+4 \beta_{M}^{(P Q)}\right)\right. \\
\left.+m_{d}\left(5 \alpha_{M}^{(P Q)}+2 \beta_{M}^{(P Q)}\right)\right]\end{array}$ & $\begin{array}{l}\frac{1}{6}\left(2 g_{A} g_{1}+g_{1}^{2}\right) \times \\
\quad\left[m_{u}\left(\alpha_{M}^{(P Q)}+4 \beta_{M}^{(P Q)}\right)\right. \\
\left.\quad+m_{d}\left(5 \alpha_{M}^{(P Q)}+2 \beta_{M}^{(P Q)}\right)\right]\end{array}$ & $\begin{array}{l}\frac{1}{12}\left(2 g_{A}+g_{1}\right)^{2} \times \\
\quad\left[m_{u}\left(\alpha_{M}^{(P Q)}+4 \beta_{M}^{(P Q)}\right)\right. \\
\left.\quad+m_{d}\left(5 \alpha_{M}^{(P Q)}+2 \beta_{M}^{(P Q)}\right)\right]\end{array}$ & \\
\hline
\end{tabular}


TABLE X: The coefficients $G_{\phi}^{B}$ and $G_{\phi \phi^{\prime}}^{B}$ in PQ $\chi \mathrm{PT}$. Coefficients are listed for the nucleons, and for $G_{\phi}^{B}$ are grouped into contributions from loop mesons with mass $m_{\phi}$, while for $G_{\phi \phi^{\prime}}^{B}$ are grouped into contributions from pairs of quark-basis $\eta_{q}$ mesons.

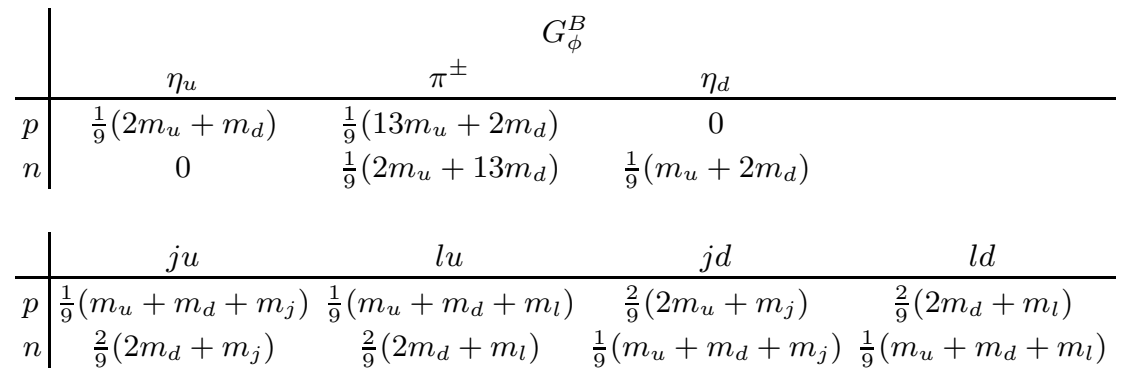

\begin{tabular}{c|ccc} 
& \multicolumn{3}{|c}{$G_{\phi \phi^{\prime}}^{B}$} \\
& $\eta_{u} \eta_{u}$ & $\eta_{u} \eta_{d}$ & $\eta_{d} \eta_{d}$ \\
\hline$p$ & $\frac{2}{9}\left(2 m_{u}+m_{d}\right)$ & $-\frac{4}{9}\left(2 m_{u}+m_{d}\right)$ & $\frac{2}{9}\left(2 m_{u}+m_{d}\right)$ \\
$n$ & $\frac{2}{9}\left(m_{u}+2 m_{d}\right)$ & $-\frac{4}{9}\left(m_{u}+2 m_{d}\right)$ & $\frac{2}{9}\left(m_{u}+2 m_{d}\right)$
\end{tabular}

TABLE XI: The coefficients $A_{\phi}^{T}, A_{\phi \phi^{\prime}}^{T}, B_{\phi}^{T}$ and $B_{\phi \phi^{\prime}}^{T}$ in PQ $\chi \mathrm{PT}$. Coefficients are listed for the delta states $T$, and for $A_{\phi}^{T}$ and $B_{\phi}^{T}$ are grouped into contributions from loop mesons with mass $m_{\phi}$, while for $A_{\phi_{\phi^{\prime}}}^{T}$ and $B_{\phi \phi^{\prime}}^{T}$ are grouped into contributions from pairs of quark-basis $\eta_{q}$ mesons.

\begin{tabular}{l|ccccccc|ccc} 
& \multicolumn{7}{|c}{$A_{\phi}^{T}$} & \multicolumn{3}{c}{$A_{\phi \phi^{\prime}}^{T}$} \\
& $\eta_{u}$ & $\pi^{ \pm}$ & $\eta_{d}$ & $j u$ & $l u$ & $j d$ & $l d$ & $\eta_{u} \eta_{u}$ & $\eta_{u} \eta_{d}$ & $\eta_{d} \eta_{d}$ \\
\hline$\Delta^{++}$ & $\frac{2}{3}$ & 0 & 0 & $\frac{1}{3}$ & $\frac{1}{3}$ & 0 & 0 & 1 & 0 & 0 \\
$\Delta^{+}$ & $\frac{2}{9}$ & $\frac{4}{9}$ & 0 & $\frac{2}{9}$ & $\frac{2}{9}$ & $\frac{1}{9}$ & $\frac{1}{9}$ & $\frac{4}{9}$ & $\frac{4}{9}$ & $\frac{1}{9}$ \\
$\Delta^{0}$ & 0 & $\frac{4}{9}$ & $\frac{2}{9}$ & $\frac{1}{9}$ & $\frac{1}{9}$ & $\frac{2}{9}$ & $\frac{2}{9}$ & $\frac{1}{9}$ & $\frac{4}{9}$ & $\frac{4}{9}$ \\
$\Delta^{-}$ & 0 & 0 & $\frac{2}{3}$ & 0 & 0 & $\frac{1}{3}$ & $\frac{1}{3}$ & 0 & 0 & 1
\end{tabular}

\begin{tabular}{c|ccccccc|ccc} 
& \multicolumn{7}{|c}{$B_{\phi}^{T}$} & \multicolumn{3}{c}{$B_{\phi \phi^{\prime}}^{T}$} \\
& $\eta_{u}$ & $\pi^{ \pm}$ & $\eta_{d}$ & $j u$ & $l u$ & $j d$ & $l d$ & $\eta_{u} \eta_{u}$ & $\eta_{u} \eta_{d}$ & $\eta_{d} \eta_{d}$ \\
\hline$\Delta^{++}$ & $-\frac{2}{3}$ & 0 & 0 & $\frac{2}{3}$ & $\frac{2}{3}$ & 0 & 0 & 0 & 0 & 0 \\
$\Delta^{+}$ & $-\frac{2}{9}$ & $-\frac{4}{9}$ & 0 & $\frac{4}{9}$ & $\frac{4}{9}$ & $\frac{2}{9}$ & $\frac{2}{9}$ & $\frac{2}{9}$ & $-\frac{4}{9}$ & $\frac{2}{9}$ \\
$\Delta^{0}$ & 0 & $-\frac{4}{9}$ & $-\frac{2}{9}$ & $\frac{2}{9}$ & $\frac{2}{9}$ & $\frac{4}{9}$ & $\frac{4}{9}$ & $\frac{2}{9}$ & $-\frac{4}{9}$ & $\frac{2}{9}$ \\
$\Delta^{-}$ & 0 & 0 & $-\frac{2}{3}$ & 0 & 0 & $\frac{2}{3}$ & $\frac{2}{3}$ & 0 & 0 & 0
\end{tabular}

TABLE XII: The coefficients $C_{\phi}^{T}, C_{\phi \phi^{\prime}}^{T}, D_{\phi}^{T}$ and $D_{\phi \phi^{\prime}}^{T}$ in PQ $\chi \mathrm{PT}$ are listed for the delta states $T$, and for $C_{\phi}^{T}$ and $D_{\phi}^{T}$ are grouped into contributions from loop mesons with mass $m_{\phi}$, while for $C_{\phi \phi^{\prime}}^{T}$ and $D_{\phi \phi^{\prime}}^{T}$ are grouped into contributions from pairs of quark-basis $\eta_{q}$ mesons. If a particular meson or pair of flavor-neutral mesons is not listed, then the value of the coefficient is zero for all deltas. The coefficients $\bar{C}_{\phi}, \bar{C}_{\phi \phi^{\prime}}, \bar{D}_{\phi}$ and $\bar{D}_{\phi \phi^{\prime}}$ are identical for all nucleons and deltas and appear in Table $\nabla I I$

\begin{tabular}{c|cccc|cc} 
& \multicolumn{5}{|c|}{$C_{\phi}^{T}$} & \multicolumn{2}{c}{$C_{\phi \phi^{\prime}}^{T}$} \\
& $j u$ & $l u$ & $j d$ & $l d$ & $\eta_{u} \eta_{u}$ & $\eta_{d} \eta_{d}$ \\
\hline$\Delta^{++}$ & $m_{u}+m_{j}$ & $m_{u}+m_{l}$ & 0 & 0 & $2 m_{u}$ & 0 \\
$\Delta^{+}$ & $\frac{2}{3}\left(m_{u}+m_{j}\right)$ & $\frac{2}{3}\left(m_{u}+m_{l}\right)$ & $\frac{1}{3}\left(m_{d}+m_{j}\right)$ & $\frac{1}{3}\left(m_{d}+m_{l}\right)$ & $\frac{4}{3} m_{u}$ & $\frac{2}{3} m_{d}$ \\
$\Delta^{0}$ & $\frac{1}{3}\left(m_{u}+m_{j}\right)$ & $\frac{1}{3}\left(m_{u}+m_{l}\right)$ & $\frac{2}{3}\left(m_{d}+m_{j}\right)$ & $\frac{2}{3}\left(m_{d}+m_{l}\right)$ & $\frac{2}{3} m_{u}$ & $\frac{4}{3} m_{d}$ \\
$\Delta^{-}$ & 0 & 0 & $m_{d}+m_{j}$ & $m_{d}+m_{l}$ & 0 & $2 m_{d}$
\end{tabular}

\begin{tabular}{c|cccc|cc} 
& \multicolumn{5}{|c|}{$D_{\phi}^{T}$} & \multicolumn{2}{|c}{$D_{\phi \phi^{\prime}}^{T}$} \\
& $j u$ & $l u$ & $j d$ & $l d$ & $\eta_{u} \eta_{u}$ & $\eta_{d} \eta_{d}$ \\
\hline$\Delta^{++}$ & 1 & 1 & 0 & 0 & 1 & 0 \\
$\Delta^{+}$ & $\frac{2}{3}$ & $\frac{2}{3}$ & $\frac{1}{3}$ & $\frac{1}{3}$ & $\frac{2}{3}$ & $\frac{1}{3}$ \\
$\Delta^{0}$ & $\frac{1}{3}$ & $\frac{1}{3}$ & $\frac{2}{3}$ & $\frac{2}{3}$ & $\frac{1}{3}$ & $\frac{2}{3}$ \\
$\Delta^{-}$ & 0 & 0 & 1 & 1 & 0 & 1
\end{tabular}


TABLE XIII: The coefficients $F_{\phi}^{T}$ and $F_{\phi \phi^{\prime}}^{T}$ in PQ $\chi \mathrm{PT}$. Coefficients are listed for the delta states $T$, and for $F_{\phi}^{T}$ are grouped into contributions from loop mesons with mass $m_{\phi}$, while for $F_{\phi \phi^{\prime}}^{T}$ are grouped into contributions from pairs of quark-basis $\eta_{q}$ mesons.

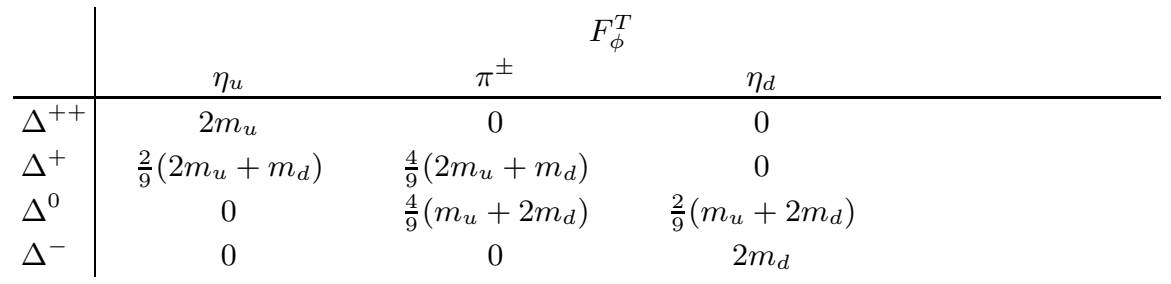

\begin{tabular}{l|cccc} 
& $j u$ & $l u$ & $j d$ & $l d$ \\
\hline$\Delta^{++}$ & $\frac{1}{3}\left(2 m_{u}+m_{j}\right)$ & $\frac{1}{3}\left(2 m_{u}+m_{l}\right)$ & 0 & 0 \\
$\Delta^{+}$ & $\frac{2}{9}\left(m_{u}+m_{d}+m_{j}\right)$ & $\frac{2}{9}\left(m_{u}+m_{d}+m_{l}\right)$ & $\frac{1}{9}\left(2 m_{u}+m_{j}\right)$ & $\frac{1}{9}\left(2 m_{u}+m_{l}\right)$ \\
$\Delta^{0}$ & $\frac{1}{9}\left(2 m_{d}+m_{j}\right)$ & $\frac{1}{9}\left(2 m_{d}+m_{l}\right)$ & $\frac{2}{9}\left(m_{u}+m_{d}+m_{j}\right)$ & $\frac{2}{9}\left(m_{u}+m_{d}+m_{l}\right)$ \\
$\Delta^{-}$ & 0 & 0 & $\frac{1}{3}\left(2 m_{d}+m_{j}\right)$ & $\frac{1}{3}\left(2 m_{d}+m_{l}\right)$
\end{tabular}

\begin{tabular}{c|ccc} 
& \multicolumn{3}{|c}{$F_{\phi \phi^{\prime}}^{T}$} \\
& $\eta_{u} \eta_{u}$ & $\eta_{u} \eta_{d}$ & $\eta_{d} \eta_{d}$ \\
\hline$\Delta^{++}$ & $3 m_{u}$ & 0 & 0 \\
$\Delta^{+}$ & $\frac{4}{9}\left(2 m_{u}+m_{d}\right)$ & $\frac{4}{9}\left(2 m_{u}+m_{d}\right)$ & $\frac{1}{9}\left(2 m_{u}+m_{d}\right)$ \\
$\Delta^{0}$ & $\frac{1}{9}\left(m_{u}+2 m_{d}\right)$ & $\frac{4}{9}\left(m_{u}+2 m_{d}\right)$ & $\frac{4}{9}\left(m_{u}+2 m_{d}\right)$ \\
$\Delta^{-}$ & 0 & 0 & $3 m_{d}$
\end{tabular}

TABLE XIV: The coefficients $E_{\phi}^{T}$ and $E_{\phi \phi^{\prime}}^{T}$ in PQ $\chi \mathrm{PT}$. Coefficients are listed for the delta states $T$, and for $E_{\phi}^{T}$ are grouped into contributions from loop mesons with mass $m_{\phi}$, while for $E_{\phi \phi^{\prime}}^{T}$ are grouped into contributions from pairs of quark-basis $\eta_{q}$ mesons.

\begin{tabular}{l|ccc|ccc} 
& \multicolumn{3}{|c|}{$E_{\phi}^{T}$} & \multicolumn{3}{|c}{$E_{\phi \phi^{\prime}}^{T}$} \\
& $\eta_{u}$ & $\pi^{ \pm}$ & $\eta_{d}$ & $\eta_{u} \eta_{u}$ & $\eta_{u} \eta_{d}$ & $\eta_{d} \eta_{d}$ \\
\hline$\Delta^{++}$ & 1 & 0 & 0 & 1 & 0 & 0 \\
$\Delta^{+}$ & $\frac{1}{3}$ & $\frac{2}{3}$ & 0 & $\frac{1}{3}$ & $\frac{2}{3}$ & 0 \\
$\Delta^{0}$ & 0 & $\frac{2}{3}$ & $\frac{1}{3}$ & 0 & $\frac{2}{3}$ & $\frac{1}{3}$ \\
$\Delta^{-}$ & 0 & 0 & 1 & 0 & 0 & 1
\end{tabular}


TABLE XV: The coefficients $G_{\phi}^{T}$ and $G_{\phi \phi^{\prime}}^{T}$ in PQ $\chi \mathrm{PT}$. Coefficients are listed for the delta states $T$, and for $G_{\phi}^{T}$ are grouped into contributions from loop mesons with mass $m_{\phi}$, while for $G_{\phi \phi^{\prime}}^{T}$ are grouped into contributions from pairs of quark-basis $\eta_{q}$ mesons.

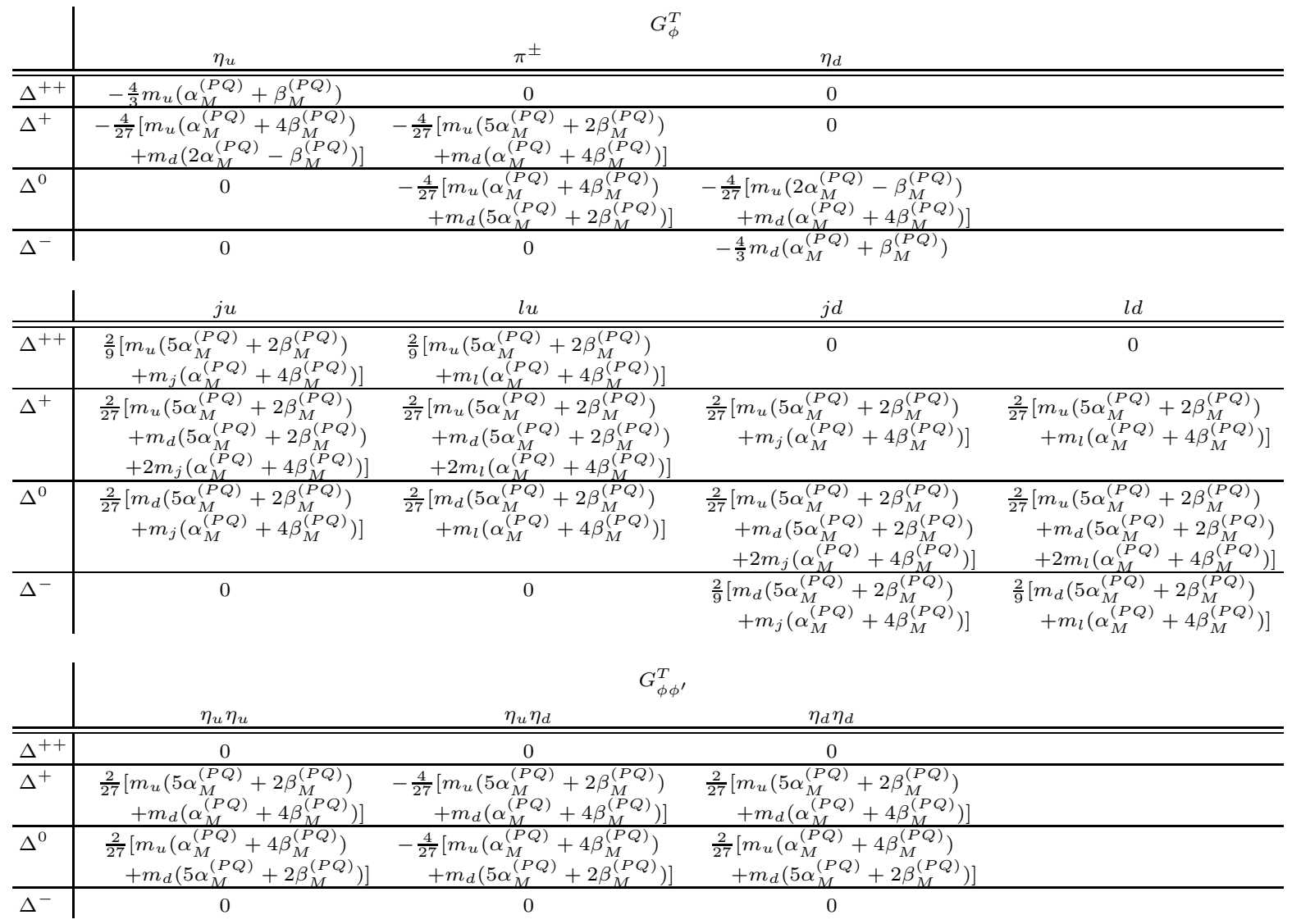

